\title{
Modeling of hydrogen isotope inventory in mixed materials including porous deposited layers in fusion devices
}

\author{
Chaofeng Sang ${ }^{1,2}$, Xavier Bonnin ${ }^{1}$, Manoj Warrier ${ }^{3}$, Abha Rai ${ }^{4}$, \\ Ralf Schneider ${ }^{5}$, Jizhong Sun ${ }^{2}$, Dezhen Wang ${ }^{2}$ \\ ${ }^{1}$ LSPM-CNRS, Université Paris 13, Villetaneuse, France \\ 2 School of Physics and Optoelectronic Technology, Dalian University of Technology, \\ Dalian, China \\ 3 Computational Analysis Division, Facility for Electromagnetic Systems, BARC, \\ India \\ ${ }^{4}$ Max-Planck-Institut für Plasmaphysik, Greifswald, Germany \\ ${ }^{5}$ Institute of Physics, Ernst-Moritz Arndt University Greifswald, Germany \\ E-mail: chaofengsang@gmail.com, bonnin@lspm.cnrs.fr
}

\begin{abstract}
.
Hydrogen isotopes inventory (HII) is a key issue for fusion devices like ITER. Simultaneous use of Be, $\mathrm{W}$ and $\mathrm{C}$ as the wall material for different parts of plasmafacing components (PFCs) will bring in material mixing issues, which compound that of hydrogen isotopes retention. To simulate the hydrogen inventory in the PFCs, we have developed a flexible standalone model called HIIPC (Hydrogen Isotope Inventory Processes Code). The particle balance based model for reaction-diffusion and HII in metal and porous media (mainly carbon and co-deposited layer) is presented, coupled with a heating model which can calculate the temperature distribution. Some sample results are given to illustrate the model's capabilities and show good qualitative agreement with experiment.
\end{abstract}




\section{Introduction}

The next-step fusion device ITER can't rely on a single material for plasma facing components, due to the very different particle and energy loads at different positions within the device [1]. Currently, the primary materials choice for the first wall of ITER is beryllium for main chamber, CFC at the strike points and tungsten at the divertor baffles and dome $[2,3]$. With the development of fusion technology, it becomes clear that Plasma Surface Interactions (PSIs) are a big issue for designing future fusion reactors because of the erosion of wall, safety reasons, etc. $[4,5]$. One of the important aspects of PSI is hydrogen isotopes (HI) retention and inventory [6].

Retention of $\mathrm{HI}$ is particularly important if the isotope is tritium. A large amount of tritium retention in the plasma-facing wall would increase the fuel costs of the reactor and presents a safety concern [6]. Therefore, understanding of the hydrogen isotope inventory processes (HIIPs) in the wall (for different materials) is extremely important; and presence of mixed materials in the fusion device makes it even more difficult because of co-deposited compounds and porosity effects $[1,5]$ (physical and chemical sputtering by fast HIs ions and charge-exchange neutral atoms causes erosion of the plasma-facing materials, deposition of one material onto another and creation of mixed co-deposited materials).

There have been several different experimental studies of HIIPs on different materials $[7,8,9,10,11]$, and some focus on different properties such as solubility and diffusivity [12]; however, HIIPs are still far from fully understood due to their complexity. Hence, it is necessary to use numerical methods to get further information of HIIPs, and thus a simulation model is required.

Different types of codes, such as Molecular Dynamics (MD) models [13], Kinetic Monte Carlo (KMC) models [14, 15, 16], and fluid codes [17, 18, 19], have been applied to study HIIPs on different issues. MD models can obtain precise information on a small time and space scale problem, and KMC can acquire information over a much larger spatio-temporal scale issue. However, the resource-consuming and scale-limited shortcomings of both MD and KMC simulations make them difficult to couple with other edge plasma codes. We want a model to give us large scale information and be flexible and fast, therefore, a fluid-based model is chosen.

The purpose of the present work is to develop a suitable standalone model, which can easily be coupled with other edge plasma codes such as the fluid-based code SOLPS [20], the particle dynamics based PIC-MCC code [21, 22, 23], and Monte-Carlo codes [24], etc., to simulate HIIPs for different wall materials including the co-deposited layer. We present here a Hydrogen Isotope Inventory Processes Code called HIIPC, in which a particle balance model for reaction-diffusion and inventory of $\mathrm{HI}$ in metals [17] and porous media based on Ref. [25] has been developed At the current stage, the model (HIIPC) includes three parts: a 1D heating module for the temperature distribution in the wall [26]; a metal module for HIIPs in metal materials (tungsten and beryllium), and a four region module for HIIPs in porous media (CFC and co-deposited layer). The goal 
of this standalone model is to provide a functional module for present fusion simulation codes and to furnish them with a capacity to take into account HIIPs in PFCs. To make the simulations reliable for the HIIPs in different materials, we need a wide variety of experimental data, such as information on trap sites, diffusivity, recombination, grain size distribution, and wall material compositions, as input for our code (this is the limitation of the current model because the shortage of input data). Hence future targeted experiments and/or $a b$ initio calculations for these data are expected.

This study is intended to show the development of our new model and its capability, and not as a prediction for fuel retention in fusion devices as coupling with edge plasma codes has not yet been completed. Relevant work is being under way.

\section{Model}

In this section, a detailed description of HIIPC is presented. The model includes three sections: heating module, metal module and porosity module.

\subsection{Heating module}

The behavior of HIIPs in the wall is highly dependent on temperature, which plays an important role in HIs retention and recycling in a fusion device. It is important to note that temperature variations can result in desorption or absorption of huge amounts of HIs compared to that in the plasma. Therefore, HIs retention and recycling studies must be done under controlled temperature conditions. The energy leaving the core plasma is deposited mostly onto the divertor plates and limiters, thereby increasing the wall temperature.

We use a heating model for the temporal evolution of the temperature distribution in the bulk wall, with a heating load $\dot{q}(t)$ at the heating side and a heat sink at the opposite side. The simulation domain is shown in Fig.1(a). The equation can be expressed as:

$$
\rho C_{p} \frac{\partial T(z, t)}{\partial t}=\frac{\partial}{\partial z}\left(k(T) \frac{\partial T(z, t)}{\partial z}\right)
$$

where $\rho$ and $C_{p}$ are the density $\left(\mathrm{kg} / \mathrm{m}^{3}\right)$ and specific heat $(J /(\mathrm{kg} \cdot K))$ of the materials, respectively, and $k(T)=1 /(a T+b)$ is the heat conductivity $(W /(m \cdot K))$, where the constants $a$ and $b$ are fitted from experiments [26]. The fitted form of $k(T)$ is used for convenience, as it allows an analytic solution at steady-state for simple cases, but is not an absolute requirement.

At the top boundary, which receives the heating flux, the boundary condition (BC) is:

$$
\left.\frac{\partial T(z, t)}{\partial z}\right|_{\text {front }}=\frac{\dot{q}(t)}{k(T)}
$$


At the back boundary, where is the cooling side with a fixed temperature $\left(T_{0}\right)$, the $\mathrm{BC}$ is:

$$
\left.T(z, t)\right|_{\text {back }}=T_{0}
$$

Combining equations 1, 2 and 3, the temperature distribution can be calculated using the finite difference method. This allows for sufficient flexibility to consider any PFC construction as a succession of layers, including coatings.

\subsection{Metal module}

Beryllium and tungsten are the most important candidates for the first wall of ITER. For safety reasons, it is important to understand HIs in these two types of metal materials. The mechanisms of penetration of HI molecular gas and atoms (ions) into the metal are different. When HIs are absorbed in the bulk of a metal, they have to pass through the surface. In order to penetrate into the bulk, a molecule needs to dissociate into two atoms. It means there are only HI atoms inside the materials. The energetic particles penetrating into the metal progressively create lattice defects in the near surface layer and deposit their energy by electron excitation, atomic displacement and phonon excitation. The randomly deposited hydrogen can become trapped in the defects of the metal.

The HIs retention concentration inside the materials can be split into two populations: solute and trapped. Four main HIIPs, all thermally activated, are involved in such metal materials: diffusion, trapping, detrapping and surface recombination to form molecules, and can be represented by a set of coupled differential rate equations $[17,18]$ :

$$
\begin{aligned}
& \frac{\partial C_{s}(z, t)}{\partial t}=\frac{\partial}{\partial z}\left(D \frac{\partial C_{s}(z, t)}{\partial z}\right)+(1-R) \Gamma_{0} \phi(z)-\frac{\partial C_{t}(z, t)}{\partial t}, 0<z<L \\
& \frac{\partial C_{t}(z, t)}{\partial t}=\frac{2 D a}{3}\left(C_{s}\left(C_{t}^{0}-C_{t}\right)-\frac{12 C_{t}}{a^{3}} \cdot \exp \left(-\frac{E_{H d t r a p}}{k_{b} T}\right)\right)
\end{aligned}
$$

where the HIs concentration $C$ is composed of a solute concentration $C_{s}$ and a trapped concentration $C_{t}$, e.g. $C=C_{s}+C_{t} ; L$ is the depth of the simulation domain; $z$ is the simulation direction which is parallel to the normal to the wall surface (see Fig. $1(\mathrm{a})) ; D=D_{0} \cdot \exp \left(-E_{m} / k_{b} T\right)$ is the diffusion coefficient $\left(m^{2} / s\right), \phi(z)$ is the HIs implantation profile, $\Gamma_{0}$ is the particle flux $\left(\right.$ atom $\left./ \mathrm{m}^{2} / \mathrm{s}\right), R$ is the backscattering coefficient which can be calculated via [27]; $a$ is the lattice constant; $C_{t}^{0}$ is the intrinsic trap site density; $T$ is the temperature and $k_{b}$ is the Boltzmann constant. In this metal module, we assume that there is no grains or voids in the metal materials wall.

The recombination processes can only happen at the surface of the wall (here the surface means plasma facing surface, see Fig.1(a)), which can be expressed as:

$$
J_{s}=K_{r} C_{s}^{2}
$$


hence, the BCs on the front and back surface are given by particle balance [17]:

$$
\left.\lambda \frac{\partial C_{s}(z, t)}{\partial t}\right|_{s}=\left.\left(-2 J_{s}+D \frac{\partial C_{s}(z, t)}{\partial z}+(1-r) \Gamma_{0} \phi(z) \lambda-\lambda \frac{\partial C_{t}(z, t)}{\partial t}\right)\right|_{s}
$$

where the subscript $s$ stands for surface, and $\lambda=a / 2 \sqrt{2}$ is the jumping distance.

There are two kinds of traps that are included in our model, intrinsic and ioninduced traps. To include ion-induced traps in the model, we use the ion-induced trap concentration [17]:

$$
C_{\text {trap }, \text { induced }}(z, t)=C_{\text {trap }, \max }\left(1-\exp \left(-(1-R) \Gamma_{0} \phi(z) \eta t / C_{\text {trap }, \text { max }}\right)\right)
$$

where $C_{\text {trap,max }}$ is the maximal defect concentration, and $\eta$ is the rate of defect production.

\subsection{Porosity module}

Carbon-based materials and co-deposited layers are made of granules and voids. To simulate HIIPs in these porous media, we develop a four-region model based on [25], the details of each region being: Region I, internal surfaces of co-deposit layer; Region II, bulk co-deposit; Region III, internal surfaces of target material; Region IV, Bulk target, see Fig. 1(b). It is a one-dimensional model along the direction normal to the plasma-facing surface (see Fig.1(a)). Time-dependent diffusion equations are used to describe the transport of the various species in different regions.

We consider five basic processes in our model:

(1) Diffusion in various regions;

A trapping-detrapping diffusion is assumed and an expression for the structure dependent diffusion coefficient as derived in [29].

(2) Interegional transport, e.g. transport from one region to another;

There exists 4 different kinds of inter-regional transport:

- Internal surface to internal surface (Region-I to Region-III and Region-III to Region-I, this processes only occur at the interface of co-deposited layer and target);

- Internal surface to bulk (Region-I to Region-II, Region-III to Region-IV );

- Bulk to Bulk (Region-II to Region-IV and Region-IV to Region-II, this processes only occur at the interface of co-deposited layer and target);

- Bulk to internal surface (Region-II to Region-I and Region-IV to Region-III)

(3) Eley-Rideal (ER) processes, wherein an incident atom recombines with an atom adsorbed onto the surface to form a molecule (note that the ER process occurs only for particles adsorbed on surfaces); By comparing the area occupied by a hydrogen atom adsorbed on the surface with the area of influence of an incoming hydrogen atom, the Eley-Rideal process can be represented.

(4) Langmuir-Hinshelwood (LH) process wherein two adsorbed atoms diffuse and recombine on the surface. By comparing the area covered by a diffusing species to the area occupied by a single particle of the specie, the LH processes can be represented; 
(5) Thermal release: thermally activated events are handled by the ArrheniusBoltzmann like relation.

All the representation of the processes described before can be found bellowing. Before giving the equations, we first introduce the symbols and conventions used: $\sigma$ indicates surface density $\left(m^{-2}\right), n$ indicates volumetric density $\left(m^{-3}\right), r$ indicates a rate per unit area per unit time, $f_{i m p, c d}$ and $f_{i m p, t}$ indicate the void fraction of grain surface in the co-deposited layer (regions I) and target (regions III). $D$ stands for the diffusion coefficient. $E$ is the migration energy of a process. $A$ stands for arrival; $H D$ means atomic desorption (here thermal desorption), $\mathrm{H}_{2} E R$ is $\mathrm{HI}$ molecule formation due to ER mechanism; $\mathrm{H}_{2} \mathrm{LH}$ means HI molecule formation due to LH mechanism. HTrap is HI trapping at an open bond site; HdTrap means detrapping of trapped HI atoms, $S D$ means surface diffusion. $R$ is the backscattering coefficient which can be calculated via [27] (same definition to metal module).

\subsubsection{Region $I$}

$$
\begin{gathered}
\frac{\partial \sigma^{I}}{\partial t}=D^{I} \frac{\partial^{2} \sigma^{I}}{\partial z^{2}}+\left(1-f_{i m p, c d}\right) r_{A}^{I}-r_{H D}^{I}-r_{H_{2} E R}^{I}-2 r_{H_{2} L H}^{I} \\
-r_{\text {HTrap }}^{I}+r_{\text {HdTrap }}^{I}+r^{I I-I}-r^{I-I I} \\
\frac{\partial \sigma_{\text {HTrap }}^{I}(z)}{\partial t}=r_{H T r a p}^{I}(z)-r_{H d T r a p}^{I}(z) \\
\sigma_{\text {Trap }}^{I}(z)=\sigma_{\text {Trap }, t=0}^{I}(z)-\sigma_{H T r a p}^{I}(z)
\end{gathered}
$$

Where $\sigma^{I}, \sigma_{\text {Htrap }}^{I}, \sigma_{\text {Trap }}^{I}, \sigma_{\text {Trap }, t=0}^{I}$ are the solute and trapped HIs densities, timedependent and initial trap sites densities in region I, respectively.

The expressions for the various reactions are:

$$
D^{I}=\omega_{0}\left(\left(L_{S D}^{I}\right)^{2} \cdot \exp \left(-\frac{E_{S D}^{I}}{k_{b} T}\right)+\left(L_{v o i d}^{I}\right)^{2} \cdot \exp \left(-\frac{E_{D}^{I}}{k_{b} T}\right)\right)
$$

where $\omega_{0}$ is the jump attempt frequency, $L_{S D}, L_{v o i d}, E_{S D}, E_{D}$ are jump length and energy requested for surface and void, respectively.

$$
\begin{aligned}
& r_{A}^{I}=\Gamma_{0}(1-R) \\
& r_{H D}^{I}=\left.\sigma^{I}\right|_{s} \omega_{0} \cdot \exp \left(-\frac{E_{H D}^{I}}{k_{b} T}\right) \\
& r_{H_{2} E R}^{I}=\left.r_{A}^{I} \sigma^{I}\right|_{s} \pi R_{0}^{2}
\end{aligned}
$$

where $R_{0}$ is the influence radius of an incoming atoms.

Rates $\left(1-f_{i m p, c d}\right) r_{A}^{I}, r_{H D}^{I}$ and $r_{H_{2} E R}^{I}$ are only taken into account at the boundary.

$$
\begin{aligned}
& r_{H_{2} L H}^{I}=\sigma^{I} \sigma^{I} \pi 2 d D^{I} \\
& r_{\text {Htrap }}^{I}=\sigma_{\text {Trap }}^{I} \sigma^{I} \pi 2 d D^{I}
\end{aligned}
$$




$$
\begin{aligned}
& r_{H d t r a p}^{I}=\sigma_{H \text { trap }}^{I} \omega_{0} \cdot \exp \left(-\frac{E_{\text {trap }}^{I}}{k_{b} T}\right) \\
& r^{I-I I}=\sigma^{I} \omega_{0} \cdot \exp \left(-\frac{E_{I-I I}}{k_{b} T}\right) \\
& r^{I I-I}=\frac{n^{I I} \sqrt{2 d D^{I I} \Delta t}}{2 \Delta t}
\end{aligned}
$$

where $d$ is the dimensionality of the region where the diffusion is happening, $\Delta t=$ 1 second (it is not the time step) which is here to show how the source term is correct dimensionally.

\subsubsection{Region $I I$}

$\frac{\partial n^{I I}}{\partial t}=D^{I I} \frac{\partial^{2} n^{I I}}{\partial z^{2}}+f_{i m p, c d} r_{A}^{I} \phi(z)-2 r_{H_{2} L H}^{I I}-\frac{S_{I}}{V_{I I}} r_{H}^{I I-I}+\frac{S_{I}}{V_{I I}} r^{I-I I}$

where $S_{I}$ is the surface area of region I, $V_{I I}$ is the volume of region II.

$$
\begin{aligned}
& D^{I I}=\omega_{0}\left(L_{\text {trap }}^{I I}\right)^{2} e^{-\frac{E_{\text {trap }}^{I I}}{k_{b} T}} \\
& r_{H_{2} L H}^{I I}=\frac{n^{I I} n^{I I} \frac{4}{3} \pi\left(2 d D^{I I} \Delta t\right)^{\frac{3}{2}}}{\Delta t}
\end{aligned}
$$

Terms $f_{i m p, c d} r_{A}^{I}$ and $2 r_{H_{2} L H}^{I I}$ are only taken into account at the boundary.

\subsubsection{Region III}

$$
\begin{gathered}
\frac{\partial \sigma^{I I I}}{\partial t}=D^{I I I} \frac{\partial^{2} \sigma^{I I I}}{\partial z^{2}}+\left(1-f_{\text {imp }, t}\right) r_{A}^{I I I}-r_{H D}^{I I I}-r_{H_{2} E R}^{I I I}-2 r_{H_{2} L H}^{I I I} \\
-r_{\text {HTrap }}^{I I I}+r_{H d T r a p}^{I I I}+r^{I V-I I I}-r^{I I I-I V}
\end{gathered}
$$

where

$$
\begin{aligned}
& \frac{\partial \sigma_{\text {HTrap }}^{I I I}(z)}{\partial t}=r_{H T r a p}^{I I I}(z)-r_{\text {HdTrap }}^{I I I}(z) \\
& \sigma_{\text {Trap }}^{I I I}(z)=\sigma_{\text {Trap }, t=0}^{I I I}(z)-\sigma_{\text {HTrap }}^{I I I}(z)
\end{aligned}
$$

The equations of each term are:

$$
D^{I I I}=\omega_{0}\left(\left(L_{S D}^{I I I}\right)^{2} \cdot \exp \left(-\frac{E_{S D}^{I I I}}{k_{b} T}\right)+\left(L_{\text {void }}^{I I I}\right)^{2} \cdot \exp \left(-\frac{E_{D}^{I I I}}{k_{b} T}\right)\right)
$$

Note $r_{A}^{I I I}=r_{A}^{I}$ when there is no co-deposited layer, when there is a co-deposited layer $r_{A}^{I I I}=0$.

$$
r_{H D}^{I I I}=\left.\sigma^{I I I}\right|_{s} \omega_{0} \cdot \exp \left(-\frac{E_{H D}^{I I I}}{k_{b} T}\right)
$$

here $r_{H D}^{I I I}=0$ when there is a co-deposited layer.

$$
r_{H_{2} E R}^{I I I}=\left.r_{A}^{I I I} \sigma^{I I I}\right|_{s} \pi R_{0}^{2}
$$


here $r_{H_{2} E R}^{I I I}=0$ when there is co-deposited layer.

Rates $\left(1-f_{i m p, t}\right) r_{A}^{I I I}, r_{H D}^{I I I}$ and $r_{H_{2} E R}^{I I I}$ are only taken into account on the surface of the wall.

$$
\begin{aligned}
& r_{H_{2} L H}^{I I I}=\sigma^{I I I} \sigma^{I I I} \pi 2 d D^{I I I} \\
& r_{H t r a p}^{I I I}=\sigma_{\text {trap }}^{I I I} \sigma^{I I I} \pi 2 d D^{I I I} \\
& r_{H d t r a p}^{I I I}=\sigma_{H \text { trap }}^{I I I} \omega_{0} e^{-\frac{E_{\text {trap }}^{I I I}}{k_{b} T}} \\
& r^{I I I-I V}=\sigma^{I I I} \omega_{0} e^{-\frac{E_{I I I-I V}}{k_{b} T}} \\
& r^{I V-I I I}=\frac{n^{I V} \sqrt{2 d D^{I V} \Delta t}}{2 \Delta t}
\end{aligned}
$$

2.3.4. Region $I V$

$$
\begin{gathered}
\frac{\partial n^{I V}}{\partial t}=f_{i m p, t} r_{A}^{I I I} \phi(z)-2 r_{H_{2} L H}^{I V}-\frac{S_{I I I}}{V_{I V}} r_{H}^{I V-I I I}+\frac{S_{I I I}}{V_{I V}} r_{H}^{I I I-I V}+D^{I V} \frac{\partial^{2} n^{I V}}{\partial z^{2}} \\
D^{I V}=\omega_{0}\left(L_{t r a p}^{I V}\right)^{2} e^{-\frac{E_{t r a p}^{I V}}{k_{b} T}} \\
r_{H_{2} L H}^{I V}=\frac{n^{I V} n^{I V} \frac{4}{3} \pi\left(2 d D^{I V} \Delta t\right)^{\frac{3}{2}}}{\Delta t}
\end{gathered}
$$

Terms $f_{i m p, t} r_{A}^{I V}$ and $2 r_{H_{2} L H}^{I V}$ are only taken into account at the boundary.

2.3.5. Boundary conditions Consider the processes which happen only in the surface, such as HIs implantation, atomic desorption and Eley-Rideal recombination, we can write the BC for grain surfaces and bulk, respectively. For region I, the BC is taken as:

$\left.\frac{\partial \sigma^{I}}{\partial t}\right|_{s}=\left.\left(\left(1-f_{i m p, c d}\right) r_{A}^{I}-r_{H D}^{I}-r_{H_{2} E R}^{I}-2 r_{H_{2} L H}^{I}-r_{H T r a p}^{I}+r_{H d T r a p}^{I}+r^{I I-I}-r^{I-I I}\right)\right|_{s}$

Region III has the same BC as region I.

For region II, the BCs can be written as

$$
\left.\frac{\partial n^{I I}}{\partial t}\right|_{s}=\left.\left(f_{i m p, c d} r_{A}^{I} \phi(z)-2 r_{H_{2} L H}^{I I}-\frac{S_{I}}{V_{I I}} r^{I I-I}+\frac{S_{I}}{V_{I I}} r^{I-I I}\right)\right|_{s}
$$

Region IV has the same BCs as region II.

On the interface of target and co-deposited layer, we assume that $\sigma^{I}=\sigma^{I I I}$ and $n^{I I}=n^{I V}$.

Surface erosion and co-deposition processes have not been fully included included in the present work. Future work will couple with the SDTrimSP [28] and WALLDYN codes $[1,30]$ to account for the wall dynamics and the chemical composition of the co-deposited layer.

In this model, a homogeneous distribution of grains inside the target and co-deposit are assumed. For carbon materials, we impose an upper limit on the maximum HIs 
volume density of $0.4 C_{\text {carbon }}[6,31]$ ( $C_{\text {carbon }}$ being the concentration of carbon atoms), meaning that when that maximal density is reached, the incoming particle flux can no longer inject additional HIs into the bulk grains until the release rate is larger than the incoming flux.

\section{Results and discussion}

We have shown the description of all the module included in the HIIPC in the above section. To give a better understanding of the HIIPs in different materials, we make some simulations using HIIPC. The simulation results are divided into three sections. The heating module is applied to simulate the wall temperature information of different materials of the wall; the metal module is used to give the HIIPs in tungsten wall; the HIIPs in carbon wall and co-deposited layer are shown via porosity module.

\subsection{The temperature distribution}

The heating module is applied here to a $\mathrm{Be} / \mathrm{C} / \mathrm{W}$ materials wall, where the heating conductivity data is obtained from [26, 27]. The wall thickness (distance of surface to the cool side) is $L$. The coolant temperature is fixed to $T_{0}=400 \mathrm{~K}$.

We use heating flux $\dot{q}=3 \mathrm{MW} / \mathrm{m}^{2}$ and $L=1 \mathrm{~cm}$. The steady-state temperature distribution inside the wall is shown in Fig. 2(a) for different materials. We can see the difference of temperature distribution between different materials, due to the difference in material properties. For carbon materials, the surface temperature can reach more than $800 \mathrm{~K}$, which is much higher than for tungsten and beryllium walls, because of the different conductivities, densities and specific heats. It takes a very short time (about $3 \mathrm{~s}$ ) for the wall temperature profile to achieve steady-state, see Fig. 2(b).

The heating flux is a very important parameter for the wall temperature. To study the effect of heating flux, we fix $L=1 \mathrm{~cm}$, and show the wall surface temperature as a function of heating flux for Be/C/W walls in Fig. 3. We can see the surface temperature increase very quickly with the heating flux, and even exceed the melting or sublimation temperature of the wall when the heating flux exceeds $10 \mathrm{MW} / \mathrm{m}^{2}$. Figure 3 can explain why the energy flux to the first wall (and divertor) of fusion device cannot be too high, usually limited to $5 \mathrm{MW} / \mathrm{m}^{2}$.

The thickness of the wall $L$ is another important parameter. We fix the heating flux $\dot{q}$ to $3 \mathrm{MW} / \mathrm{m}^{2}$. Figure 4(a) shows us the surface temperature as a function of $L$. We can see the larger $L$ is, the higher the wall surface temperature is. The carbon wall can reach its sublimation temperature when $L \geq 3 \mathrm{~cm}$; and the thickness of beryllium is also limited to $<3 \mathrm{~cm}$; tungsten is better, which does not reach its melting point until $L=6 \mathrm{~cm}$. Fig. $4(\mathrm{~b})$ shows the minimum time to achieve steady-state temperature as a function of $L$, indicating that a larger $L$ would need more time to reach steady-state temperature. For Be/C/W materials, when $\dot{q}$ and $L$ are the same, the one which can get the highest surface temperature, takes the most time to get steady-state, see Fig. 
$4(\mathrm{~b})$.

\subsection{Hydrogen isotope retention in metal wall}

Here, we apply the metal module to HIs retention in a tungsten wall. The diffusivity and trap site data is taken from [17], and recombination coefficients from [6]. The averaged injection particle energy is $20 \mathrm{eV}$ and the incidence angle with the normal to the wall surface is 60 degrees, which is applied to calculate the backscattering rate $R$. To simplify the model, we assume the HIs implantation profile $\phi(z)$ is homogeneously distributed in the surface of the wall (one spatial grid depth $(0.1 \mu m)$ ). The depth (domain) of the simulated tungsten wall is $100 \mu \mathrm{m}$.

The wall temperature is very important for HIIPs, because the diffusivity, recombination, trapping and detrapping rates all depend exponentially on it. We expose the tungsten wall to a HIs plasma fluence of $\Gamma_{0}=10^{24}$ atoms. $\mathrm{m}^{-2} . \mathrm{s}^{-1}$ for $50 \mathrm{~s}$. The retained HIs areal density as a function of wall temperature (from 350 to $1000 \mathrm{~K}$ ) is shown in Fig. 5(a). The total and solute retention HIs decrease as the temperature is increasing, however, the trapped HIs areal density first increases with temperature, and reaches its maximum $\left(1.34 \times 10^{21}\right.$ atoms. $\left.\mathrm{m}^{-2}\right)$ when the wall temperature is 410 $\mathrm{K}$, and then starts to decrease. Fig.5(b) is the percentage of trapped and solute HIs for different wall temperatures. The trapped percentage first increases with temperature, and reaches its maximum $(99.8 \%)$ at $600 \mathrm{~K}$, and then decreases. From this we find that most of the HIs are immobilized by the trap sites inside the wall in the wall temperature range $450-900 \mathrm{~K}$.

The recombination rate $K_{r}$, which controls the release of HIs, would exponentially increase with the wall temperature; therefore, the high temperature releases more HIs, and less HIs are retained. It can explain the decrease in total HIs retention amount decrease with the increasing wall temperature. The released HIs come from solute HIs inside the wall, which is the reason that the areal density of solute HIs keeps dropping when the wall temperature increases. The trapping and detrapping processes both increase with the wall temperature, the increase in trapping is the dominant processes when the temperature is below $410 \mathrm{~K}$, hence the trapped HIs density increases until $410 \mathrm{~K}$; when the temperature exceeds $410 \mathrm{~K}$, the increase in the detrapping rate becomes dominant, therefore, the trapped HIs amount start to decrease at $410 \mathrm{~K}$.

The depth profile is also important and can give detailed information about how the HIs are retained inside the wall. Figure 6 shows us HIs retention depth profiles for different wall temperatures $(400 K, 600 K, 800 K, 1000 K)$, where the surface of the wall, at $z=0$, receives the implanted HIs particles. It is shown that the depth profile is characterized by a sharp near-surface peak concentration, and a concentration tail into the bulk wall. The HIs concentration tends to diminish with the wall temperature. We can see that at $400 \mathrm{~K}$, the most HIs are retained inside the wall, however, HIs do not penetrate very deeply; at $1000 \mathrm{~K}$, HIs density is lower but extends deeper than at lower temperatures. The highest HIs density exists at the surface because of the ion-induced 
trap sites. As we explained before, lower temperatures can decrease the recombination rate $K_{r}$, thus holding more HIs inside the wall, which is the reason why there are the most HIs inside the wall at $400 K$ in Fig. 6. Higher wall temperatures increase the diffusivity which can make the HIs travel deeper into the bulk wall, explaining finding more HIs deeper in the wall when the wall temperature is higher.

We can see the importance of wall temperature during HIIPs after the above discussion. The results discussed above are all from the case with fixed wall temperature, however, in fact the temperature is not unchanged. To make the model more accurate, we couple the metal module with the heating module which can calculate the wall temperature evolution. Note that because the simulated domain of the metal module is very small $(100 \mu \mathrm{m})$ compared with the domain of the heating module $(1 \mathrm{~cm})$, we only take the surface temperature from the heating module as the wall temperature in the metal module. A direct comparison of HIs retained as function of time between fixed temperature and coupled case is shown in Fig. 7. We can see that for the fixed temperature case, the HIs areal density keeps increasing with time; however, the coupled case has different behaviour in that the HIs areal density first increases and reaches a maximum value at $t \approx 1 \mathrm{~s}$, then starts dropping till $t \approx 5 \mathrm{~s}$, before finally starting to increase again and tending to the same curve as for the fixed temperature case.

The HIs retained peak exists in the coupled case in the first $5 \mathrm{~s}$ because of the wall temperature changing. The first increase is very simple because the "empty" wall is initially charging up due to the HIs flux, and then the inventory drops because of the increasing temperature (see in insert-graph of Fig. 7 the evolution of wall temperature from the heating model). After the temperature settles, the HIs areal density increases again slowly as the HIs diffuse deeper into the bulk, making room near the surface. From the above discussion, we know that it is very important to consider the temperature evolution when the simulation time is shorter than $5 \mathrm{~s}$. We recover here an important feature distinguishing HIs retention behaviour between short and long pulse tokamak discharges, whereas during short pulses the wall acts like a pump but saturates after a few seconds of plasma exposure. Unless specified otherwise, the next set of results will use the wall temperature as computed here from the heating model.

The implanted HIs flux $\Gamma_{0}$ is another important parameter for HIIPs [5, 17]. The retention increases slightly faster than the square root of the fluence. Note that because of the low solubility and strong trapping, a small change of the trap site concentration can strongly influence the HIs retention in tungsten walls [17]. The depth profiles of HIs retained density after $50 \mathrm{~s}$ exposure under different $\Gamma_{0}\left(10^{20}, 10^{22}\right.$, $10^{24}, 10^{25}, 10^{26}, 10^{27}$ atoms. $m^{-2} s^{-1}$ ) are shown in Fig. 8. We can see very clearly that the increasing implanted HIs flux can increase the HIs retention, which is in good agreement with [5, 17]. For the depth profiles, another important phenomenon is that the larger flux can make HIs diffuse deeper inside the wall. We can see that, from $\Gamma_{0}=10^{24}$ atoms. $m^{-2} s^{-1}$, there is a small saturated region near the surface of the wall where the trap sites are all occupied by HIs. We know HIs can be very easily immobilized by trap sites, however, there are no empty trap sites in the saturated layer, therefore, 
solute HIs can diffuse deeper under high implanted HIs flux.

We have discussed HIIPs while the implanting flux is impinging on the wall, however it is also important to know what happens after the implanting flux is turned off. Exposing the tungsten wall to our implanting HIs flux $\left(10^{24}\right.$ atoms. $\left.\mathrm{m}^{-2} \mathrm{~s}^{-1}\right)$ for $50 \mathrm{~s}$, then turning off the flux and keeping the temperature unchanged, the time-evolution of the HIs retained areal density is shown in Fig. 9(a), it is clear that the HIs retention keeps dropping, however, the dropping rate also decreases with time. Figure 9(b) shows the depth profiles of HIs retention density at different times. We can see the peak of the curve moving away from the wall surface, and the HIs keep diffusing to the deeper wall. We know after the injected flux being off, there is no source and only sinks for the HIs inside the wall, thus the HIs retention keeps dropping. The solute HIs can keep diffusing after implanted flux being off. HIs in the position where the density is high would diffuse to the place where HIs density is low, thus most of the HIs would diffuse deeper inside the wall. At the plasma facing surface region, the main HIs release mechanism is recombination processes, which can decrease the HIs density. This can explain why the HIs retention near the surface keeps decreasing and in deeper regions keeps increasing. This is again an important qualitative result for tokamak wall behaviour, as it evidences the deep diffusion of HIs into the bulk of the PFCs after the end of the plasma discharges, as driven by the thermal and particle flux cycling during mahcine operation.

\subsection{Hydrogen isotope retention in porous materials}

Carbon-based materials and co-deposited layers are made of granules and voids. In this section, the porosity module is applied to simulate these porous media. The simulation target is made of carbon material, and the co-deposited layer is co-deposited carbon. Here the effect of hydrocarbons in the co-deposited layer is not taken into consideration. In this work, we do not want to directly compare the simulation results with experimental results because of the shortage of input data for material properties (especially for co-deposited layer and trap sites information) and some of the input data are artificial; however, the qualitative analysis of the simulation results can show us some very important phenomena and give us physical insight to optimize experiments. The input data used include diffusivity, trap sites, etc. and are shown in Table 1 and Table 2, where most of these were used in MD or KMC simulations $[14,15,16,25]$ and gleaned from either experiments $\left(E_{D}, E_{H D}\right.$ or an educated guess was used (like $\omega_{o}$ corresponds to typical phonon frequency [19]). The backscattering rate $R$ is from [27]. The simulated domain of the target is $3 \mu \mathrm{m}$, the thickness of the co-deposit is $2 \mu \mathrm{m}$, and incident HIs flux $\Gamma_{0}=10^{24}$ atoms. $m^{-2} \cdot s^{-1}$, unless stated otherwise. Note that in all the following figures, $z<0$ is the target layer, $z>0$ is the co-deposited layer, $z=0$ is the interface between target and co-deposited layer.

Exposing the wall to the incoming flux for $0.01 \mathrm{~s}$, at a wall temperature of $1200 \mathrm{~K}$, Fig. 10(a) presents the HIs density profiles $\left(\sigma^{I I I}, \sigma_{\text {Htrap }}^{I I I}, n^{I V}\right)$ for the two-region case (no 
co-deposit), and Fig. 10(b) shows the HIs density profiles $\left(\sigma^{I}, \sigma_{\text {Htrap }}^{I}, n^{I I}, \sigma^{I I I}, \sigma_{\text {Htrap }}^{I I I}\right.$, $n^{I V}$ ) for the four-region (carbon and co-deposited layer) case. For the two-region case, there is no co-deposited layer and the incoming flux is directly implanted into the target wall; for the four-region case, the incoming flux is implanted into the co-deposited layer. We can see that HIs can diffuse much deeper inside the co-deposit (more than $2 \mu \mathrm{m}$, Fig. 10(b)) than inside the target (less than $0.6 \mu m$, Fig. 10(a)) in this period, which can be also seen from the HIs density suddenly dropping at the interface $(z=0)$ in Fig. 10(b). The maximum density, which appears in the particle-facing region, in the two-region case (about $10^{25}$ atoms. $\mathrm{m}^{-3}$ ) is larger than in the four-region case (about $10^{22}$ atoms. $\mathrm{m}^{-3}$ ). We find a saturated layer for trapped HIs, where the surface trap sites are completed occupied (we assume the surface trap site density is $10^{19} \mathrm{~m}^{-2}$ ), in both cases; however, in the two-region case, the saturated region is larger than in four-region case.

With a wall temperature of $1200 \mathrm{~K}$, the diffusivity of the co-deposited layer $\left(\sim 0.6 \times 10^{-10} \mathrm{~m}^{2} \mathrm{~s}^{-1}\right)$ is much larger than that of the target $\left(\sim 1.2 \times 10^{-14} \mathrm{~m}^{2} \mathrm{~s}^{-1}\right)$, thus, HIs can diffuse much deeper inside the co-deposited layer than inside the target in the same time. The recombination rate (LH processes) in region II is much larger than in region IV; therefore, the maximum volume density $\left(n^{I I}\right)$ is smaller in region II (Fig. 10(b)) than the maximum $n^{I V}$ in region IV (Fig. 10(a)). (Note that the largest release rate happens in the surface of the target or co-deposit, which faces the incoming plasma, and the release flux can balance the implanted flux, thus a smaller recombination rate would cause a larger density, see equations 16 and 23).

In porous media, the temperature is also important for HIs inventory processes (similar to metal materials). Figure 11 shows us the depth profiles of retained HIs in regions I-IV, after exposing the wall to the incoming flux for $1 \mathrm{~s}$ for different wall temperatures. We can see that, the higher wall temperature is, the deeper HIs can diffuse. At $800 \mathrm{~K}$, the HIs cannot diffuse into the target at all (only existing inside the co-deposited layer), however, when the temperature is as high as $1200 \mathrm{~K}$, the HIs can diffuse into the target for more than $1 \mu \mathrm{m}$ in $1 \mathrm{~s}$. In Fig. 11(b), we can see that the values of $n^{I I}$ on the plasma-facing surface region is more than three orders of magnitude higher at $700 \mathrm{~K}$ than at $1200 \mathrm{~K}$, which means that the lower temperature can keep more HIs within the surface region of region II; however, on the surface region of region I, the lower temperature cannot make the retained HIs density higher, because it is already saturated.

The HIs diffusivity inside the wall is very sensitive to temperature, for example: in region II, the diffusivity is $\sim 1.5 \times 10^{-14} \mathrm{~m}^{2} \mathrm{~s}^{-1}(700 \mathrm{~K})$ and $\sim 0.6 \times$ $10^{-10} \mathrm{~m}^{2} \mathrm{~s}^{-1}(1200 \mathrm{~K})$; in region IV, the diffusivity is $\sim 1.9 \times 10^{-22} \mathrm{~m}^{2} \mathrm{~s}^{-1}(700 \mathrm{~K})$ and $\sim 1.2 \times 10^{-14} \mathrm{~m}^{2} \mathrm{~s}^{-1}(1200 \mathrm{~K})$. Therefore, higher temperature makes for larger diffusivity, leading the HIs to diffuse deeper inside the materials. The larger diffusivity also can increase the recombination rate, which can reduce $n^{I I}$ in the plasma-facing surface region.

We now couple the porosity model with the heating model. Note that because 
the simulated domain of the porosity module is very small $(5 \mu m)$ compared with the heating module $(1 \mathrm{~cm})$, we only take the surface temperature from the heating module as the wall temperature of the porosity module. The evolution of the HIs retained density profiles of regions II and IV $\left(n^{I I}\right.$ and $\left.n^{I V}\right)$, and regions I and III $\left(\sigma^{I}+\sigma_{\text {Htrap }}^{I}\right.$ and $\left.\sigma^{I I I}+\sigma_{\text {Htrap }}^{I I I}\right)$ is shown in Fig. 12, when the heating flux is $5 M W$ and implanted HIs flux remains unchanged. The HIs diffuse very fast inside the co-deposited layer (in less than $1 \mathrm{~s}$ ), however, the diffusion speed drops dramatically inside the target. In Fig. $12(\mathrm{a})$, we can see a density $\left(n^{I I}\right)$ peak existing at the surface of the co-deposit $(z=2 \mu \mathrm{m})$ at the beginning, and $n^{I I}(z=2 \mu m)$ keep decreasing with time to a constant in about $5 \mathrm{~s}$, the total retained HIs amount keeps increasing from $5 \mathrm{~s}$ to $10 \mathrm{~s}$. This is almost the same as the results for HIs inventory in metal discussed in the previous section, which can prove the validation of our porosity model.

We have discussed that HIs diffusion speed is dependent on the diffusivity, thus HIs diffuse faster in the co-deposited layer than in the target. The wall surface temperature would keep increasing to about $1200 \mathrm{~K}$ in $5 \mathrm{~s}$ (see inset-graph of Fig. 12(a)), while a lower temperature can increase $n^{I I}$ near the plasma facing surface, which is the reason of the density peak in Fig. 12(a). After $5 \mathrm{~s}$, the wall surface temperature stays constant, causing $n^{I I}(z=2 \mu m)$ to become almost constant after $5 \mathrm{~s}$, and the continuing diffusion of HIs deeper into the wall increases the total retained amount.

The HIs inventory, unsurprisingly, increases while an incident flux is present onto the wall. However, the HIs inventory behaviour inside the wall after that the flux is turned off is also very important for reactor safety reasons. Keeping the four-region wall exposed to the HIs flux for $1 s$, the evolution of the HIs atoms release rate after the flux being off is shown in Fig. 13, for different wall temperatures $(800-1300 \mathrm{~K})$. It can be seen that the HIs release rate drops very quickly just after the flux turning off (less than $0.01 \mathrm{~s}$ ), and then the drop rate tends to be smaller (the release rate decreases slowly). We can also see that high temperatures have a higher release rate: the release rate at $1200 \mathrm{~K}$ is about four orders of magnitude higher than at $800 \mathrm{~K}$.

The release of HIs is directly from solute HIs inside the wall. When the implanted flux is turned off, most of the solute HIs atoms inside the wall release due to LH processes, decreasing the solute HIs density; therefore, the release rate decreases dramatically. As the solute HIs atoms decrease, detrapping processes inside the wall become dominant, turning the trapped HIs into solute, and the new detrapped HIs atoms are released due to LH recombination processes. At higher temperature, the detrapping rate is larger, which means more HIs atoms are converted to solute atoms from detrapping, while the recombination rate is also larger, therefore, a larger release rate exists when the wall temperature is higher. With this mechanism, the trapped HIs density decreases, and thus the HIs release rate keeps dropping. If the time is long enough, the HIs release rate goes to zero (at constant $T$ ), and some trapped HIs are retained inside the wall. From the above discussion, we know that raising the wall temperature can be applied to clean the retained HIs inside the wall. This result is consistent with current experimental practice, for example the decrease in outgassing with time after a plasma discharge and 
the partial recovery of the HIs inventory from raising the wall temperature or baking.

To test the ability of the porosity module for dealing with the dynamic evolution of the co-deposited layer, we simulate the HIIPs while the thickness of the co-deposited layer is growing. Here we only show a simple case, with a fixed grow rate $\left(10^{-1} \mu m . s^{-1}\right)$ given while the HIs flux is on, and the initial thickness of co-deposited layer is $0.5 \mu \mathrm{m}$. Further work would couple other edge plasma and wall dynamics simulation tools with our present model. To make the analysis simple, here we use a fixed wall temperature $(800 \mathrm{~K})$. Figure 14 presents the time evolution of HIs retained density in (a) regions I and III, and (b) regions II and IV. During the growth of the co-deposited layer, the thickness of highest retained HIs density region in region I $\left(\sigma^{I}+\sigma_{\text {Htrap }}^{I}\right)$ keeps increasing (Fig. 14(a)), however, the thickness of the region with maximal $n^{I I}$ does not increase with time (Fig. 14(b)). From Fig. 14 we can see the total inventory amount would increase greatly during the growth of the co-deposited layer.

The thickness of the region with maximum $\left(\sigma^{I}+\sigma_{\text {Htrap }}^{I}\right)$ keeps increasing because most HIs retained in region I are trapped HIs $\left(\sigma_{\text {Htrap }}^{I}\right)$, and the trap sites are all occupied during the co-deposited layer growth. A higher HIs density $n^{I I}$ inside the wall would increase the recombination rate (LH processes), thus, the HIs retained density $n^{I I}$ is kept in balance with the incoming flux and the region of maximum $n^{I I}$ does not increase with time.

\section{Conclusions}

A rate model is applied to simulate HIIPs in metal materials and in a four-region description of porous media (including carbon and co-deposit layers), using input from a heating module for the temperature evolution. We reproduce qualitatively experimental behaviour of HIs retention in fusion devices,such as wall pumping, the effect of baking, HIs co-deposition and inventory growth. Further work to refine the model includes gathering of experimental data or ab initio calculations to provide the many needed parameters in the model. Coupling to other codes for dealing with the plasma response to the HIs wall inventory and to follow changes in the physico-chemical properties of the wall material mixture is being considered and will be reported in future work.

\section{Acknowledgments}

This work is funded in part by contracts ANR-09-BLAN-0070-01 (CRWTH) and CEA TechnoFusion V3580.001. C. Sang, J. Sun and D. Wang acknowledge financial support from the Chinese National Basic Research Program under grant Nos. 2008CB717801 and 2009GB106002. C. Sang would like to thank EFDA-ITM Gateway for the computational resource. 


\section{References}

[1] Ch. Linsmeier, M. Reinelt, K. Schmid, J. Nucl. Mater. (article in press) doi:10.1016/j.jnucmat.2010.08.056.

[2] J. Roth, E. Tsitrone, A. Loarte, et al., J. Nucl. Mater. 390-391, 1 (2009)

[3] J. Roth, E. Tsitrone, T. Loarer, V. Philipps, S. Brezinsek, A. Loarte, G. F. Counsell, R. P. Doerner, K. Schmid, O. V. Ogorodnikova and R. A. Causey, Plasma Phys. Control. Fusion 50103001 (2008).

[4] G. Federici, G. H. Skinner, J. N. Brooks, J. P. Coad, C. Grisolia, A.A. Haasz, et. al., Nucl. Fusion 411967 (2001).

[5] V. Kh. Alimov, H. Nakamura, B. Tyburska-Tuschel, O. V. Ogorodnikova, J. Roth, K. Isobe, T. Yamanishi, J. Nucl. Mater. 414, 479 (2011).

[6] R. A. Causey, J. Nucl. Mater. 300, 91 (2002).

[7] K. Sugiyama, M. Mayer, V. Rohde, M. Balden, Th. Durbeck, A. Herrmann, A. Wiltner, H. W. Muller, R. Neu et. al., Nucl. Fusion 50035001 (2010).

[8] M. Overkofler, M. Reinelt, A. Allouche, S. Lindig and Ch. Linsmeier, Phys. Src. T138 014036 (2009).

[9] R. A. Anderl, R. A. Causey, J. W. Davis, B. P. Doerner, G. Dederici, A. A. Haasz, et. al., J. Nucl. Mater 2731 (1999).

[10] T. Hino, F. Hirano, Y. Yamauchi, Y. Hirohata. Fusion Eng. Des. 49-50 213 (2000).

[11] E. Serra, G. Benamati, O. V. Ogorodnikova, J. Nucl. Mater 255105 (1998).

[12] R. Frauenfelder, J. Vac. Sci. Technol 6388 (1969).

[13] K. Heinola and T. Ahlgren, Phys. Rev. B 81073409 (2010).

[14] M. Warrier, R. Schneider, E. Salonen, and K. Nordlund, Nucl. Fusion 411656 (2007).

[15] R. Schneider, A. Rai, A. Mutzke, M. Warrier, E. Salonen, and K. Nordlund, J. Nucl. Mater. 367 $1238(2007)$.

[16] A. Rai, M. Warrier, R. Schneider, Comput. Mater. Sci. 46469 (2009).

[17] O. V. Ogorodnikova, J. Roth, M. Mayer, J. Nucl. Mater. 313-316, 469 (2003).

[18] O. V. Ogorodnikova, J. Nucl. Mater. 390-391, 651 (2009).

[19] A. A. Haasz, P. Franzen, J. W. Davis, S. Chu, and C. S. Pitcher, J. Appl. Phys 7766 (1995).

[20] R. Schneider, X. Bonnin, K. Borrass, et al., Contrib. Plasma Phys. 46, 3 (2006).

[21] C. Sang, J. Sun, D. Wang. Plasma Phys. Control. Fusion 52042001 (2010).

[22] C. Sang, J. Sun, and D. Wang, Fusion Eng. Des., 85, 1941 (2010).

[23] C. Sang, J. Sun, D. Wang. J. Nucl. Mater. (article in press) doi:10.1016/j.jnucmat.2011.01.007.

[24] S. Dai, J. Sun, C. Sang and D. Wang, Contributions to Plasma physics (article in press) doi:10.1002/ctpp.201000101.

[25] M. Warrier, A. Rai, R. Schneider, J. Nucl. Mater. 390-391, 203 (2009).

[26] R. Behrisch, J. Surf. Invest-X-Ray+, 4, 549 (2010).

[27] M. Warrier, R. Schneider, X. Bonnin, Compt. Phys. Comm. 160, 46 (2004).

[28] A. Rai, A. Mutzke, R. Schneider, Nucl. Instr. Meth. B 268, 2639 (2010).

[29] M. Warrier, R. Schneider, E. Salonen, and K. Nordlund, J. Nucl. Mater. 337-339, 580 (2005) .

[30] M. Reinelt, K. Krieger, S. Lisgo, K. Schmid, S. Brezinsek, JET EFDA Contributors, J. Nucl. Mater. (article in press) doi:10.1016/j.jnucmat.2010.12.246.

[31] A. A. Haasz, P. Franzen, J. W. Davis, S. Chiu and C. S. Pitcher, J. Appl. Phys. 77, 66 (1995). 


\section{Figure captions}

Figure 1: (a) Schematic of the simulation domain: the fluxes (energy and particles) are injected to the top side, and the cooling side is at the bottom, the wall is made of metal or porous media; (b) the four regions of plasma facing material.

Figure 2: (a) Steady-state temperature distribution inside the wall $(\mathrm{z}=0$ is the heating surface of the wall), (b) time-dependent surface temperature, for different materials.

Figure 3: For wall thickness (distance of surface to the cool side) $L=1 \mathrm{~cm}$, variation of the steady-state surface temperature with the heating flux.

Figure 4: For fixed heating flux $\dot{q}=1.0 \mathrm{MW} / \mathrm{m}^{2}$, (a) variation of the steady-state surface temperature with $L$, (b) minimum time to achieve steady-state temperature for different $L$ values.

Figure 5: HIIs as functions of wall temperature after exposition to a HIs flux $\Gamma_{0}=10^{24}$ atoms. $m^{-2} s^{-1}$ for $50 \mathrm{~s}$, (a) the total, solute, and trapped HIs retention; (b) the percentage of solute and trapped HIs.

Figure 6: After exposition to the HIs flux $\Gamma_{0}=10^{24} \mathrm{~m}^{-2} \mathrm{~s}^{-1}$ for $50 \mathrm{~s}$, the depth profiles of HIs retention for different wall temperatures.

Figure 7: Comparing the total HIs retained, varying with time, using either a fixed wall temperature or the temperature from our heating model. The insert graph is the temperature evolution of the two cases.

Figure 8: Depth profiles of HIs retained in the wall after $50 \mathrm{~s}$ as a function of impinging HIs flux $\Gamma_{0}$.

Figure 9: After pre-exposure to HIs flux $\Gamma_{0}=10^{24}$ atoms. $m^{-2} \cdot s^{-1}$ for $50 \mathrm{~s}$, and turning off the particle flux, (a) HIs retained as a function of time; (b) HIs retention depth profiles at different times.

Figure 10. The depth profiles of HIs retained in region I - IV: (a) two-region case, (b) four-region case. Note that $z=0$ is the interface of target and co-deposited layer.

Figure 11. Four-region case, at different temperatures $(700-1200 K), t=1 \mathrm{~s}$, the depth profiles of HIs retained in (a) $\sigma^{I}+\sigma_{\text {Htrap }}^{I}$ and $\sigma^{I I I}+\sigma_{H t r a p}^{I I I}$, (b) $n^{I I}$ and $n^{I V}$.

Figure 12. Spatial distribution evolution of (a) $n^{I I}$ and $n^{I V}$, (b) $\sigma^{I}+\sigma_{\text {Htrap }}^{I}$ and $\sigma^{I I I}+\sigma_{\text {Htrap }}^{I I I}$, when coupling to the heating module.

Figure 13. After the implanting flux $\left(\Gamma_{0}\right)$ is turned off, the HIs release rate evolution for different wall temperatures $(800-1300 \mathrm{~K})$.

Figure 14. Given a co-deposited fixed growth rate $\left(10^{-1} \mu \mathrm{m} . \mathrm{s}^{-1}\right)$, the HIs retained density distribution evolution (a) $\sigma^{I}+\sigma_{\text {Htrap }}^{I}$ and $\sigma^{I I I}+\sigma_{H t r a p}^{I I I}$, (b) $n^{I I}$ and $n^{I V}$. 
Figure 1 (a)

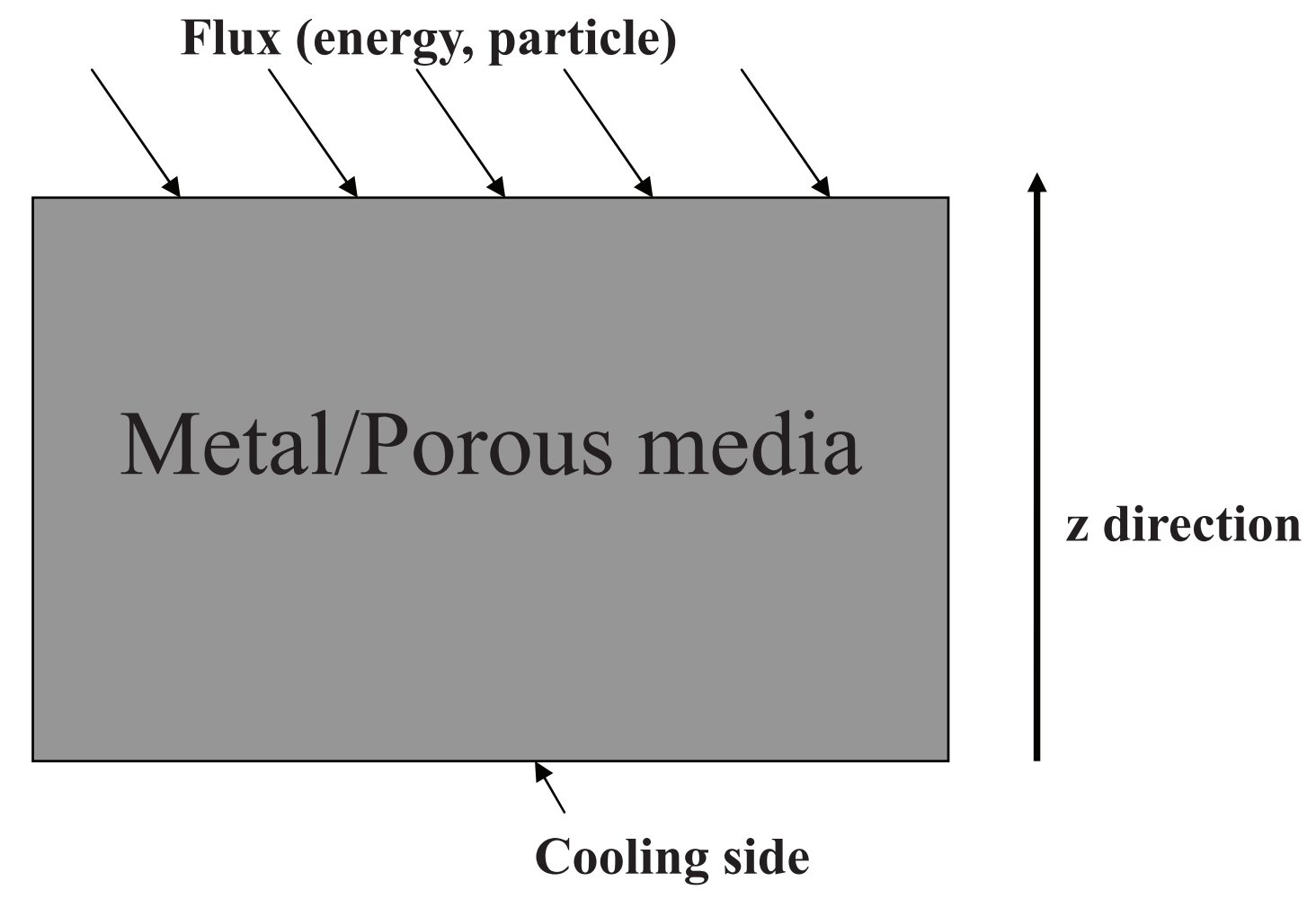


Figure 1 (b)

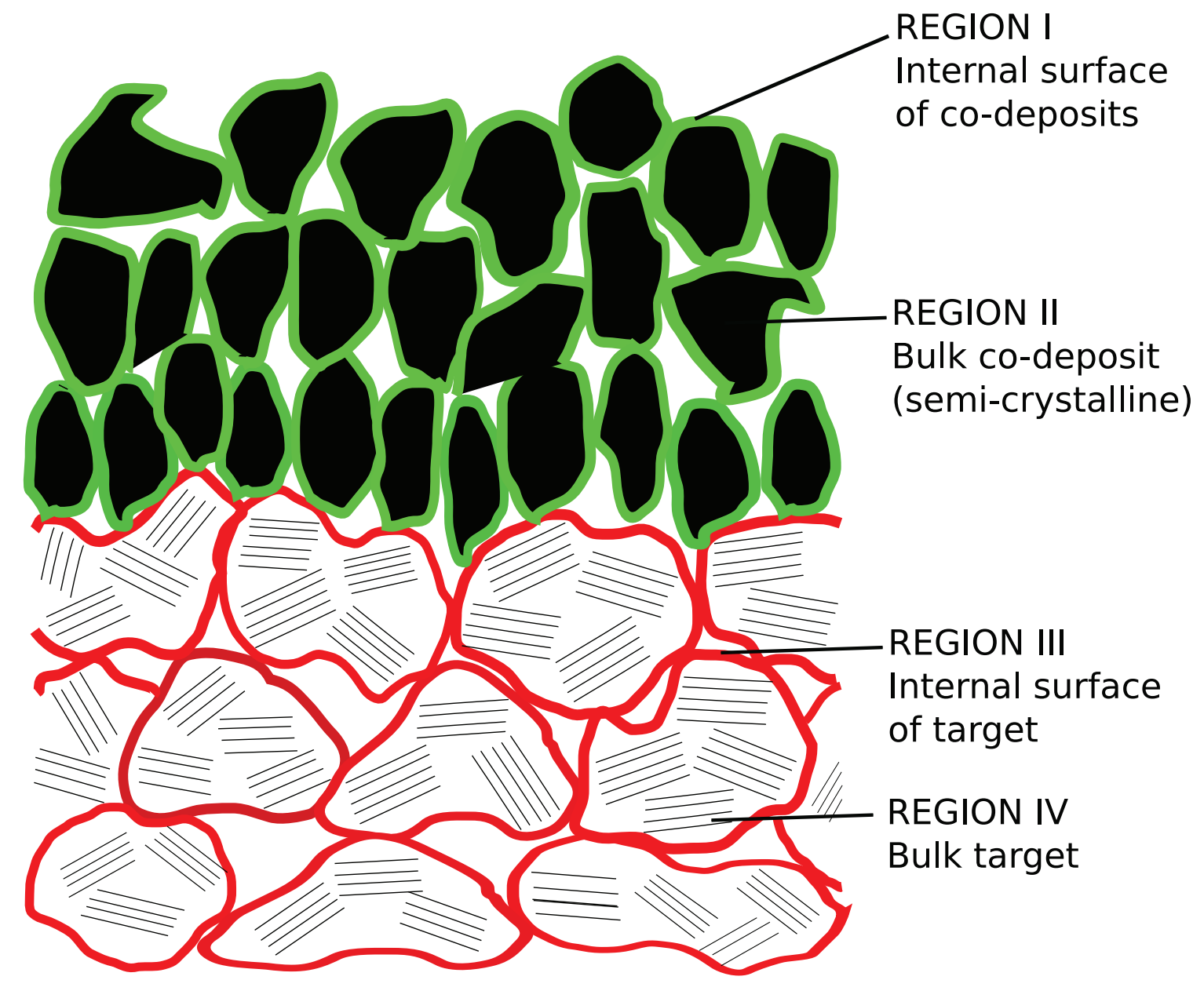


Figure 2

Heating flux: $3 \mathrm{MW} / \mathrm{m}^{2}$, cool side temperature $400 \mathrm{~K}$
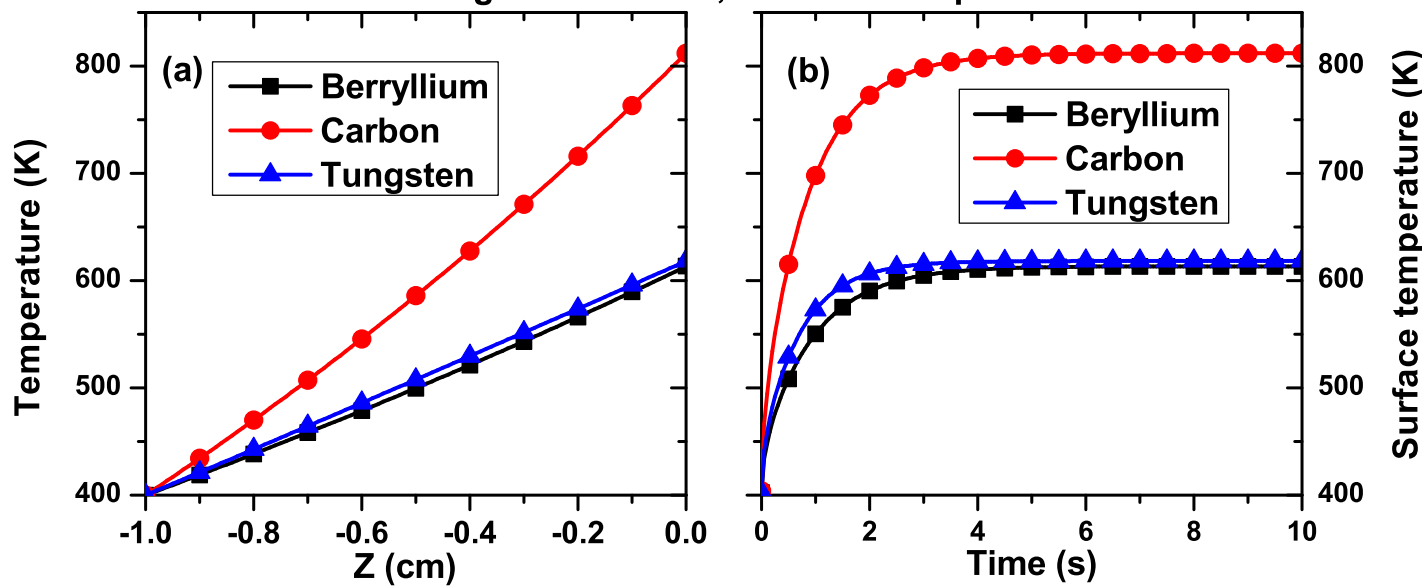
Figure 3

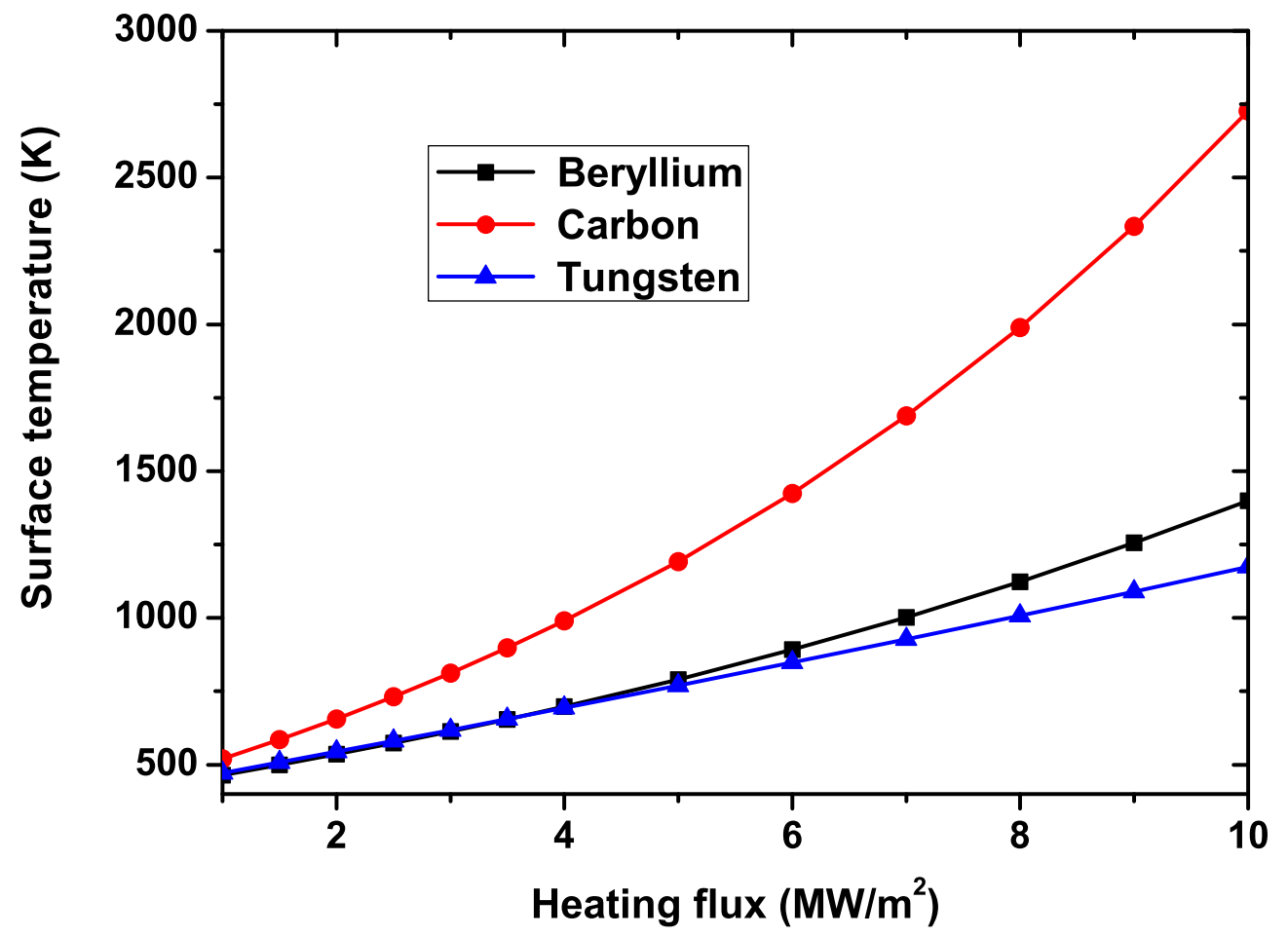


Figure 4

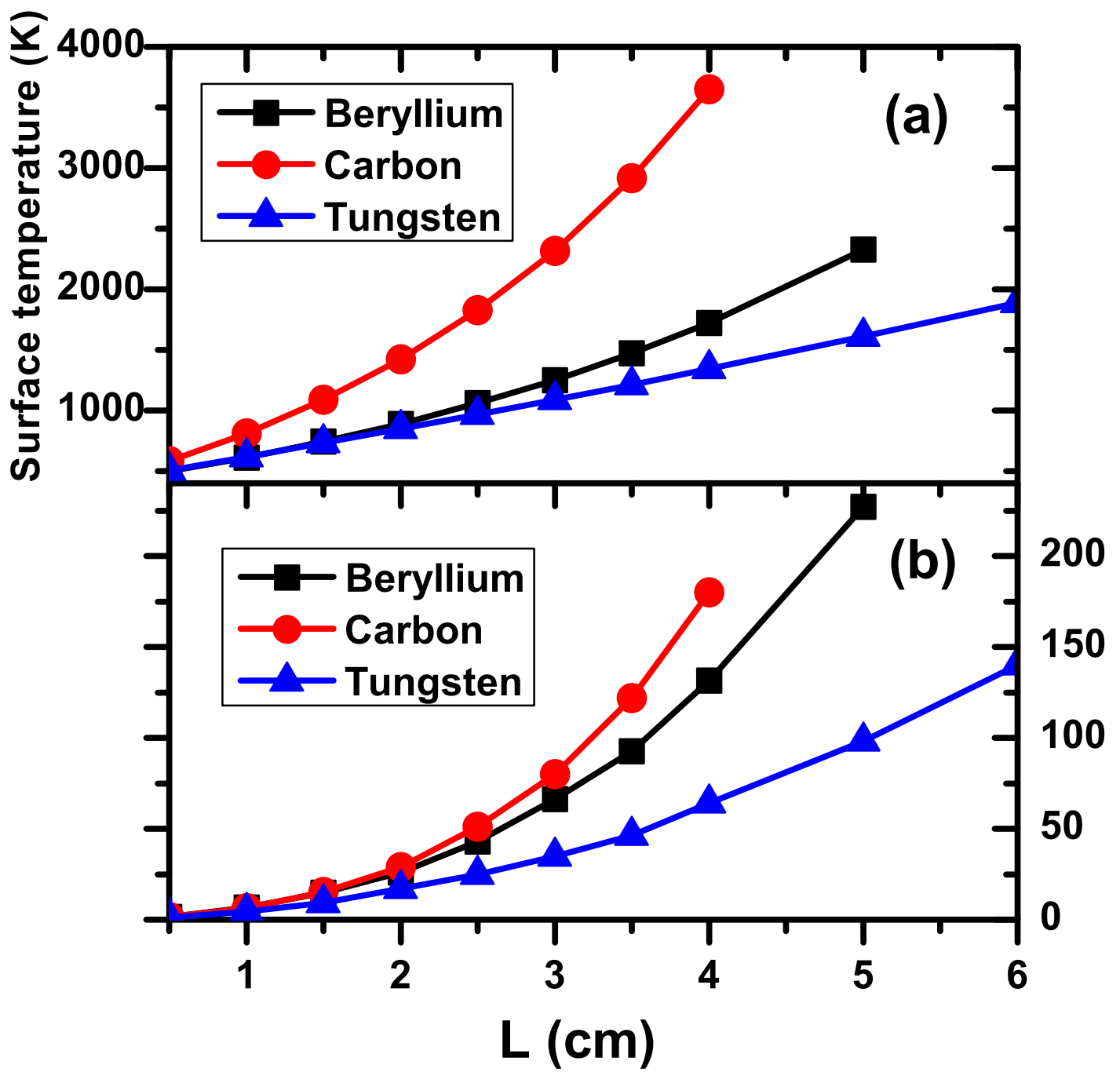

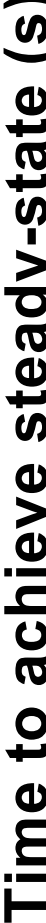


Figure 5

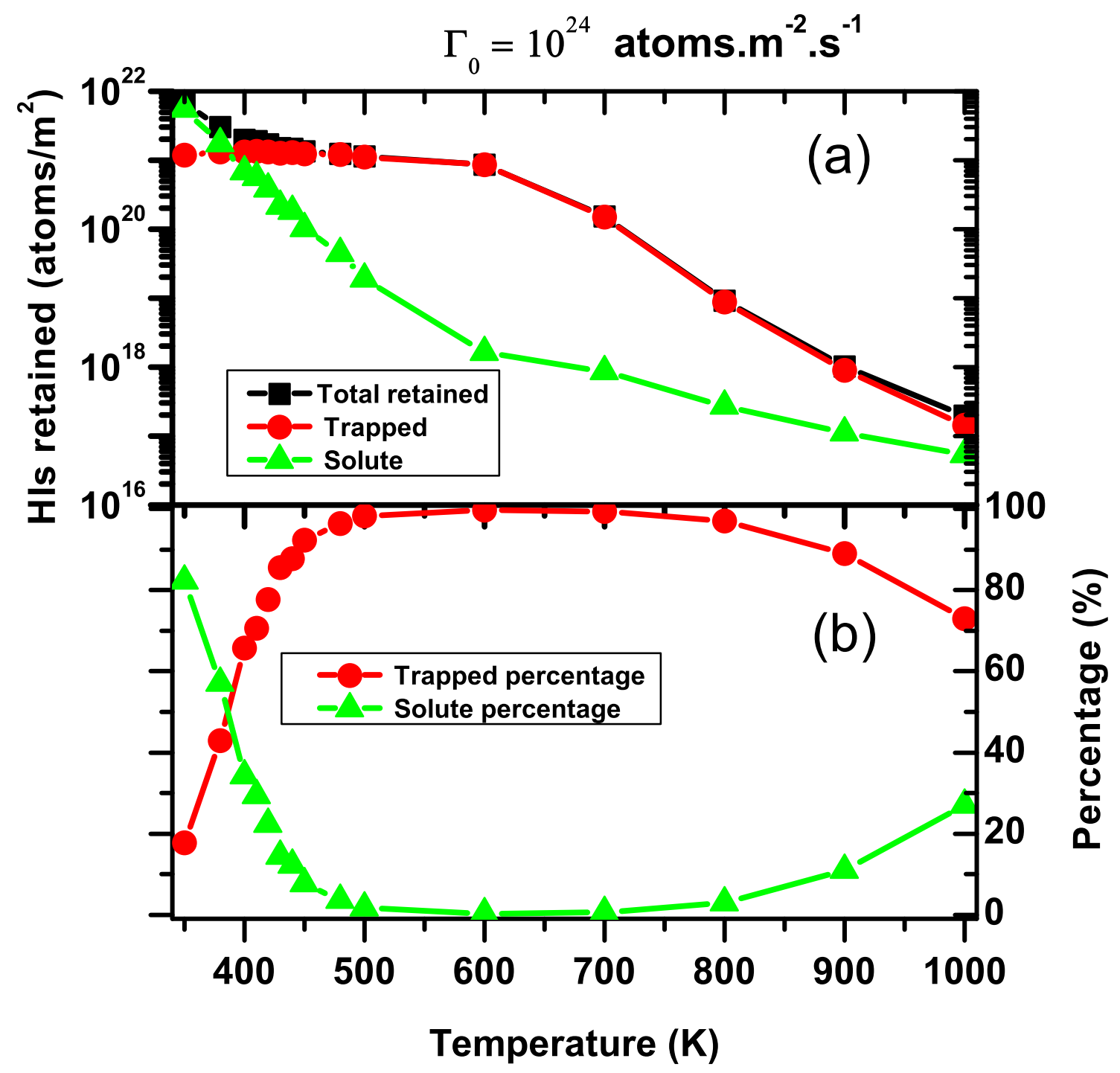


Figure 6

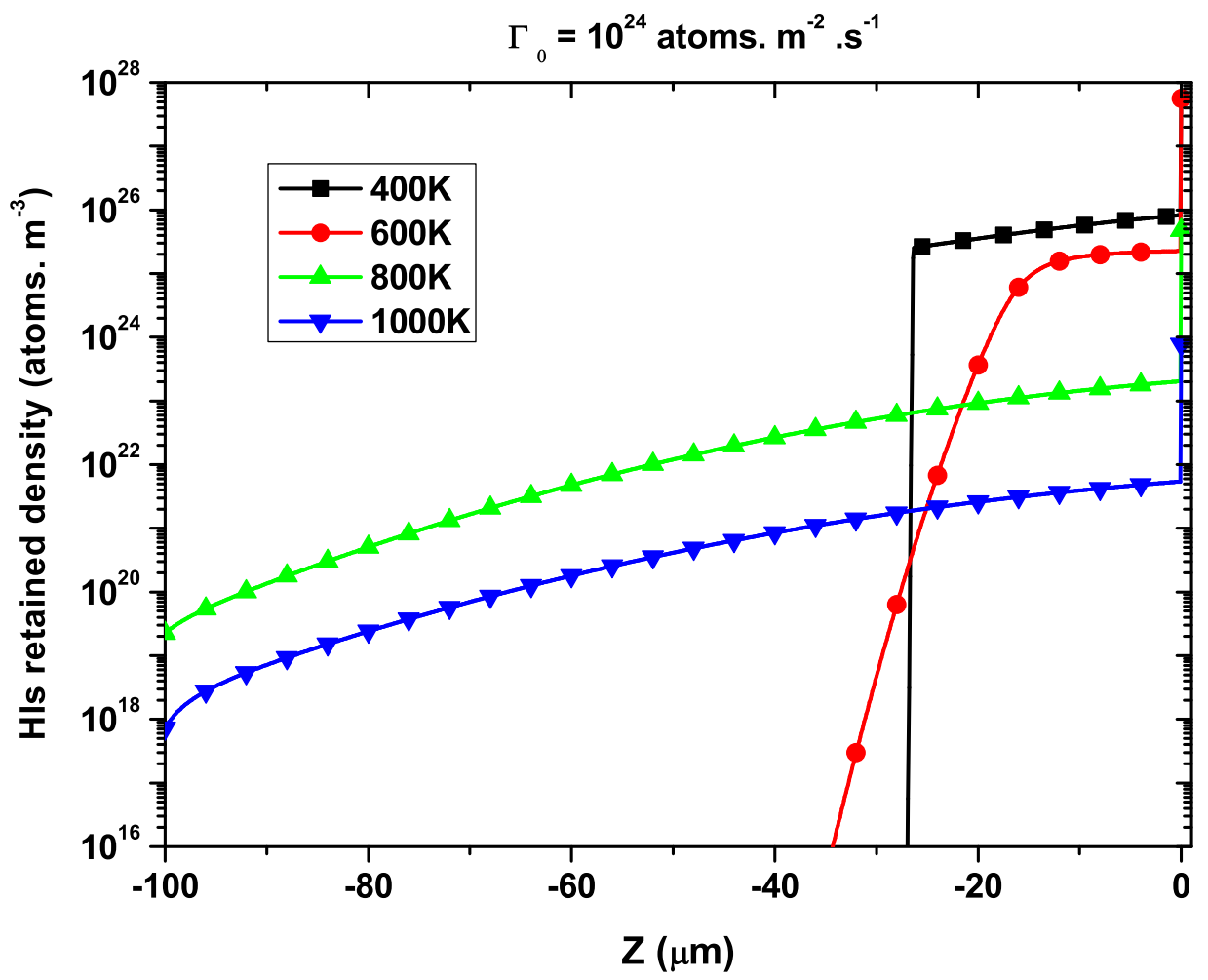


Figure 7

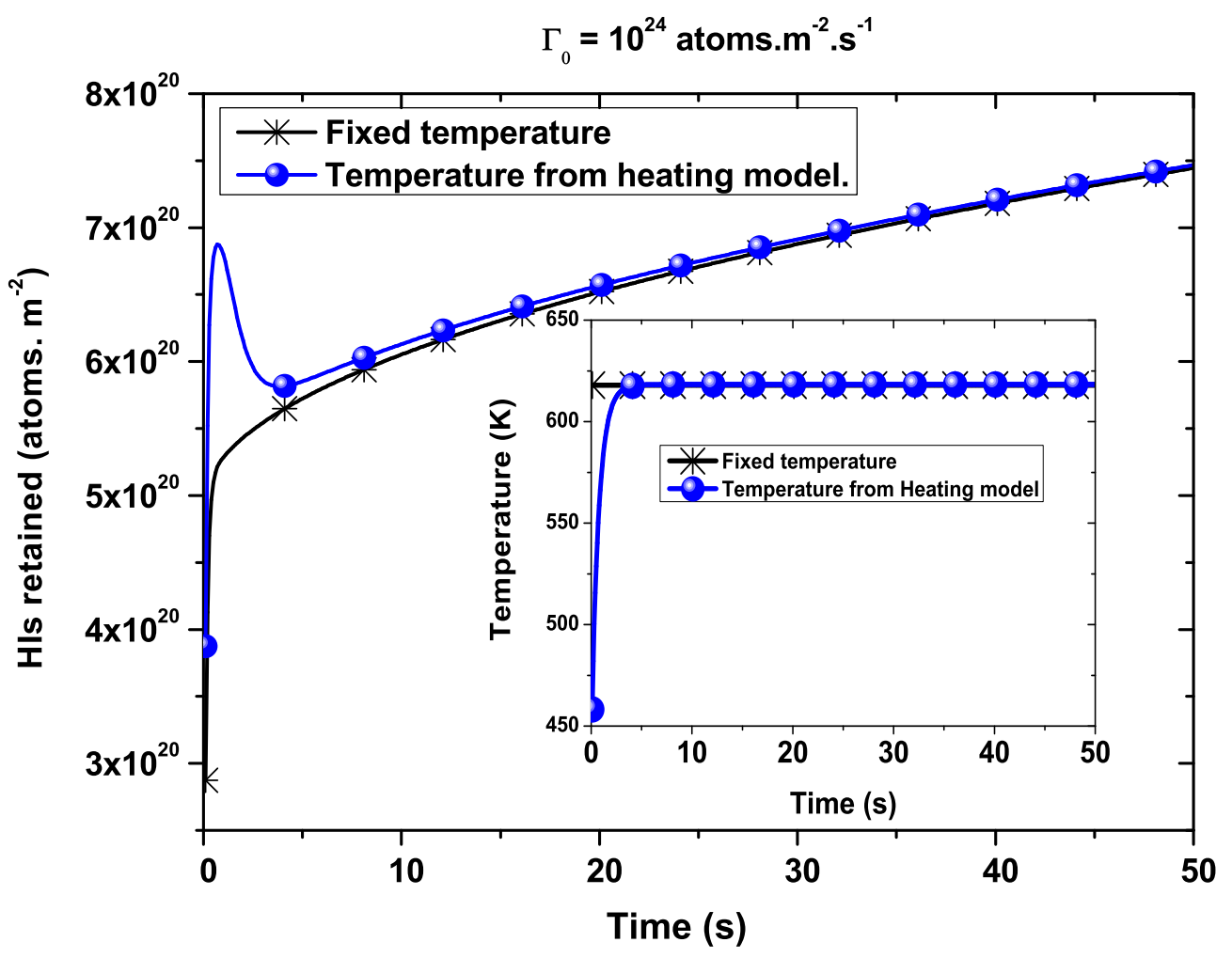


Figure 8

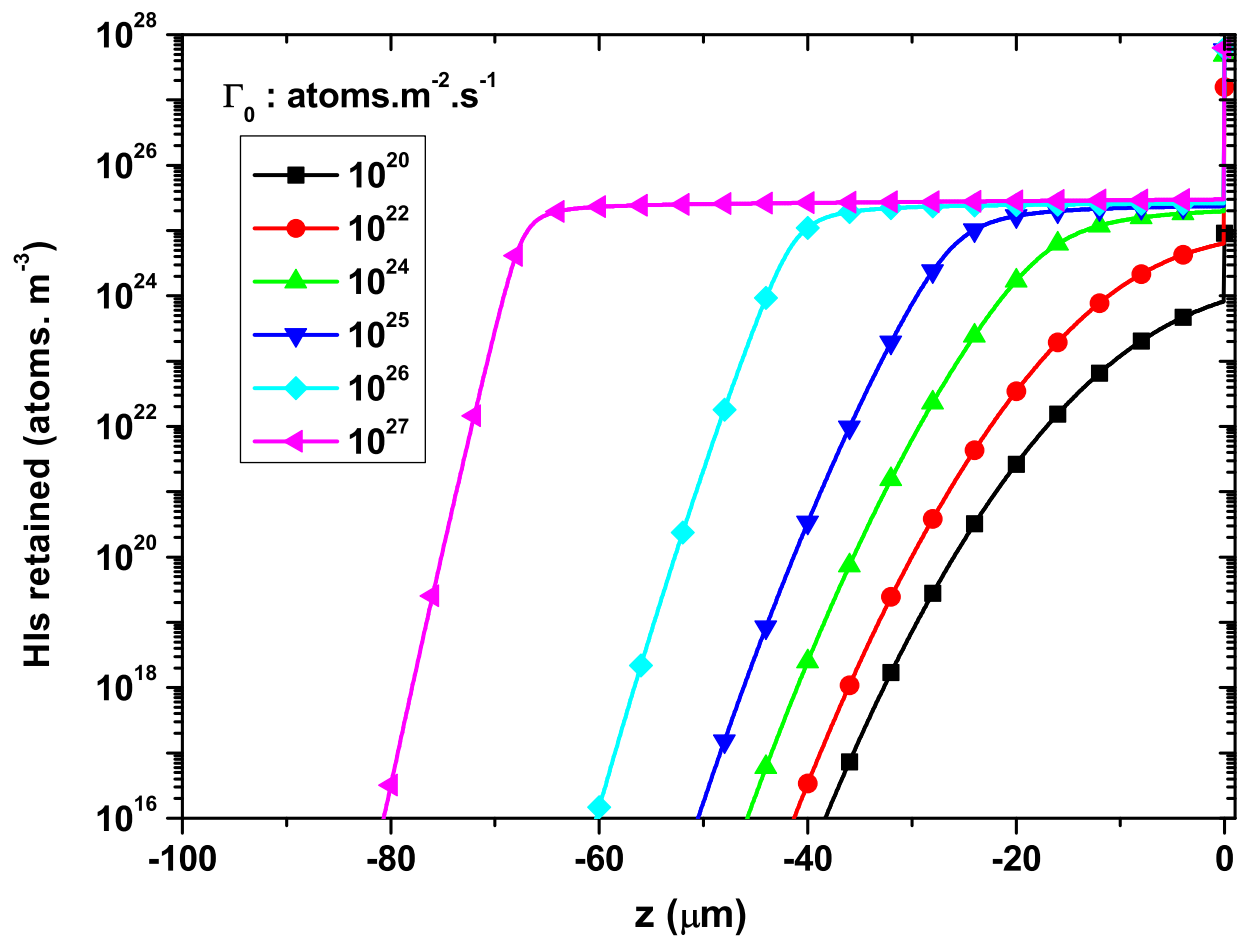


Figure 9
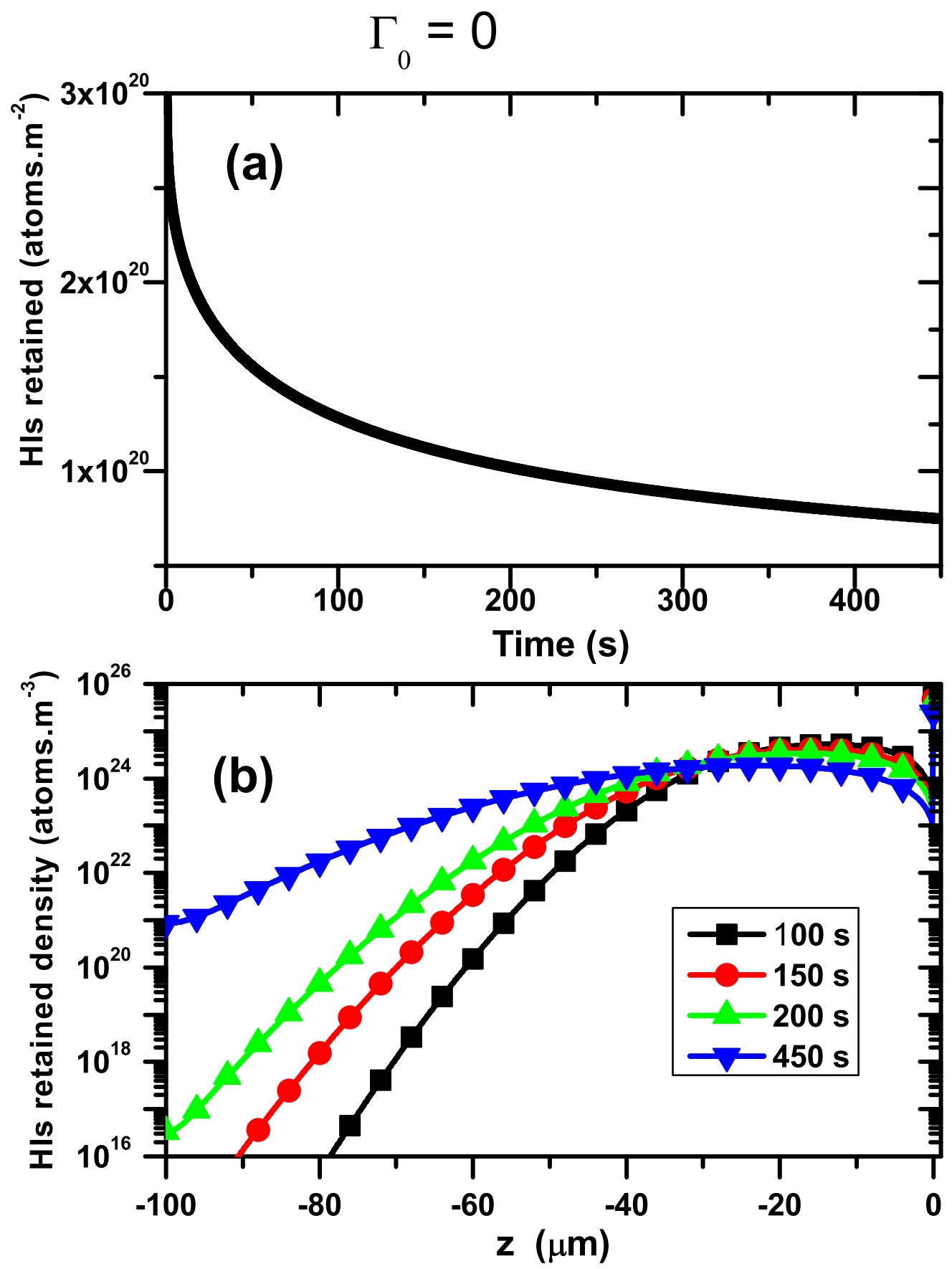
Figure 10

Temperature $1200 \mathrm{~K}, \Gamma_{0}=10^{24}$ atoms $\cdot \mathrm{m}^{-2} \cdot \mathrm{s}^{-1}$, time $=0,01 \mathrm{~s}$

(a) Two-region: target

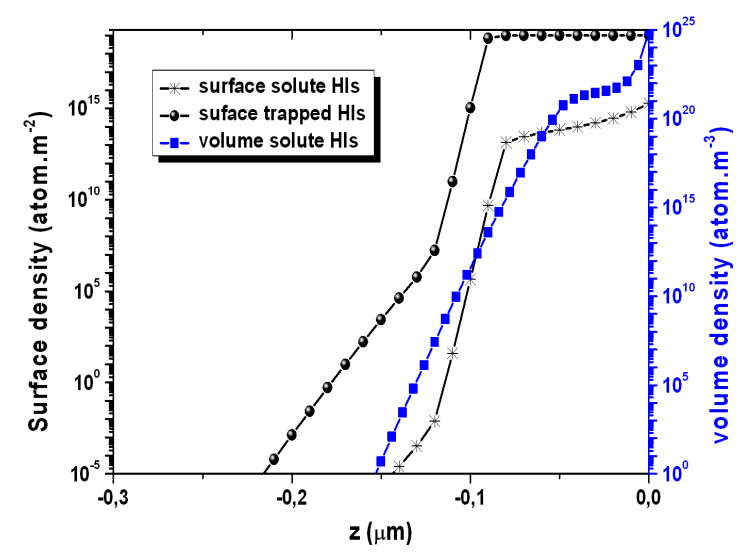

(b) Four-region: target and co-deposited layer

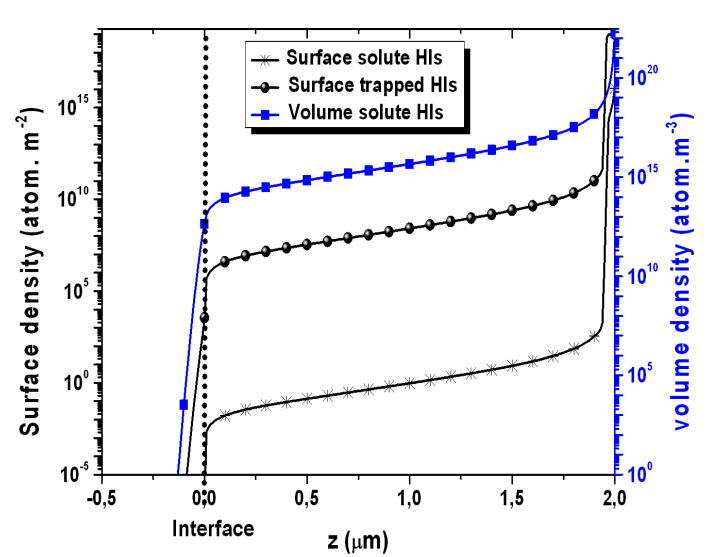


Figure 11

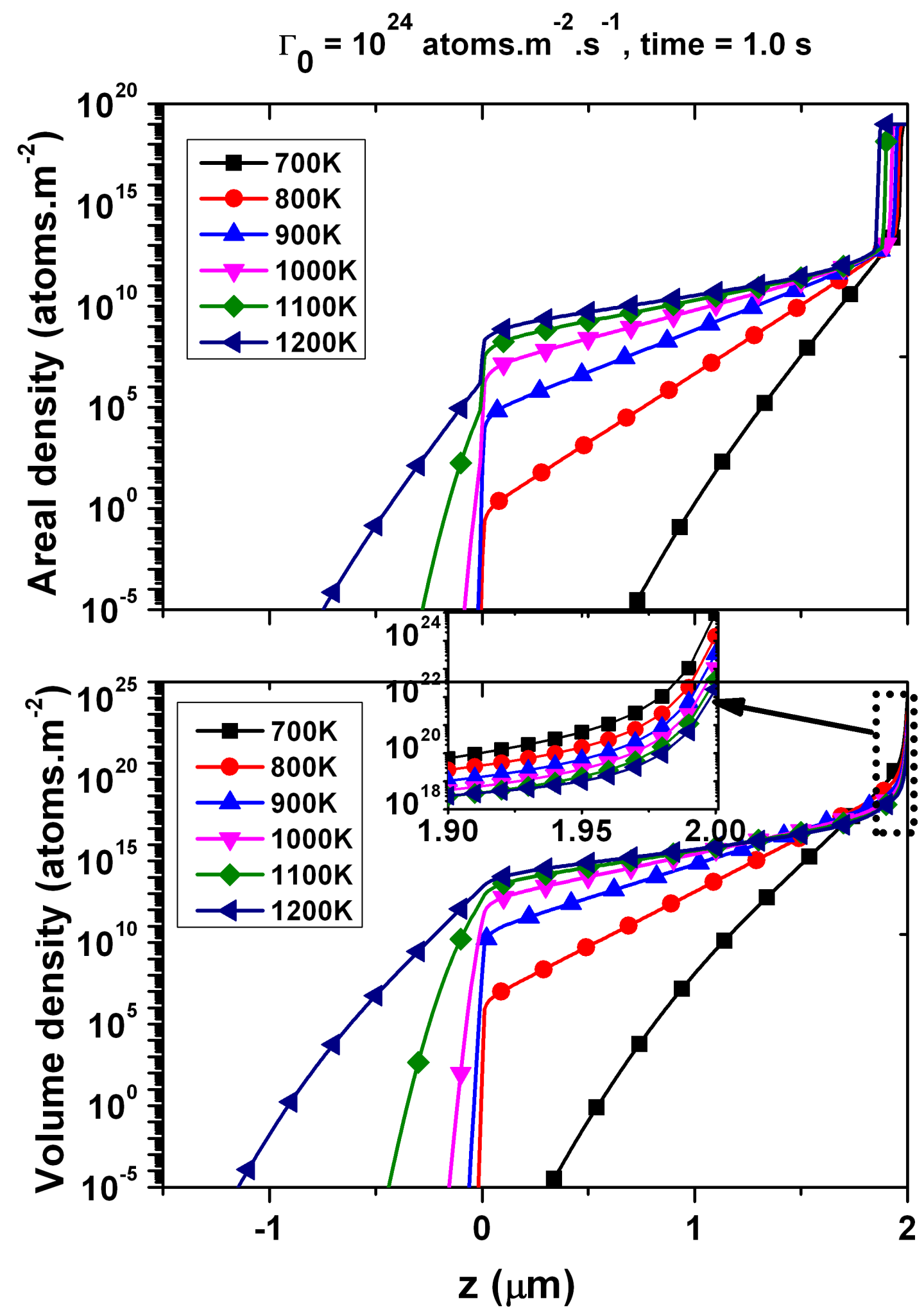

(a)

(b) 
Figure 12

Heating flux: $5 \mathrm{MW}, \Gamma_{0}=10^{24}$ atoms $\cdot \mathrm{m}^{-2} \cdot \mathrm{s}^{-1}$

(a)

(b)
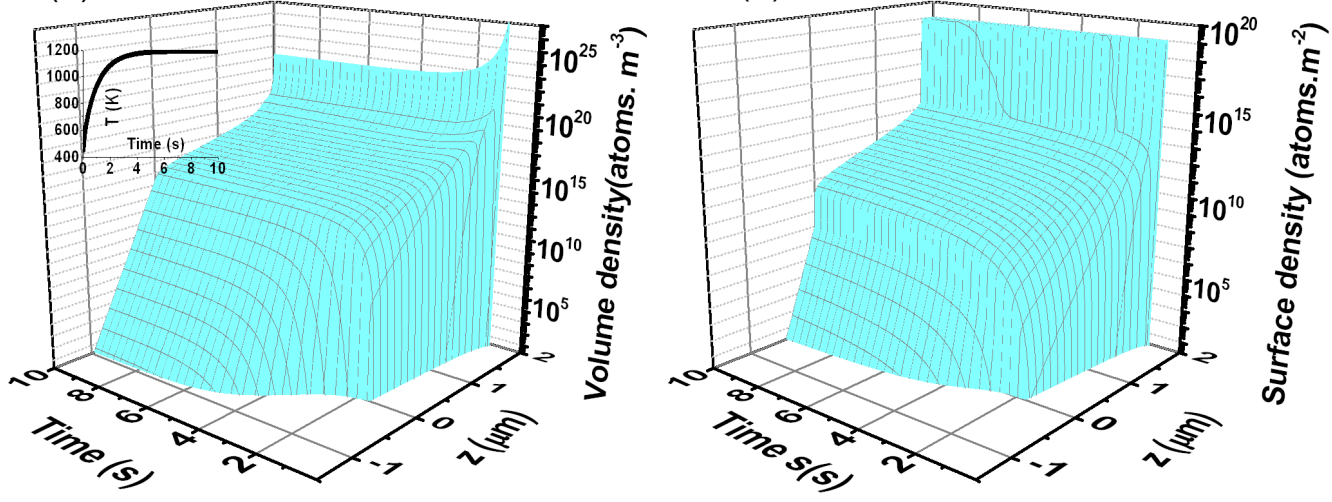
Figure 13

Exposed to $\Gamma_{0}=10^{24}$ atoms. $\mathrm{m}^{-2} \cdot \mathrm{s}^{1}$ for $1 \mathrm{~s}$. After turning off the flux, the release evolution

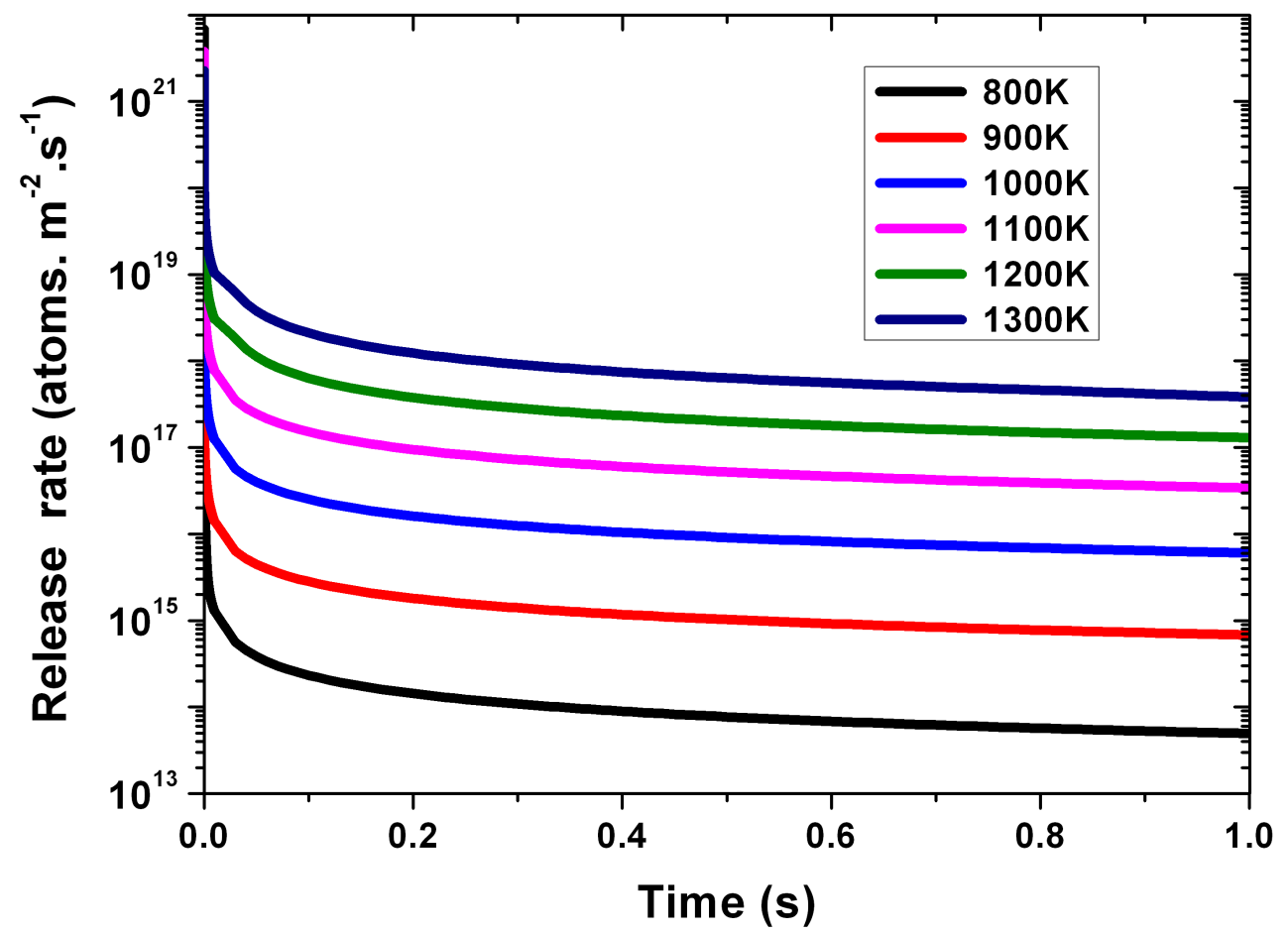


Figure 14

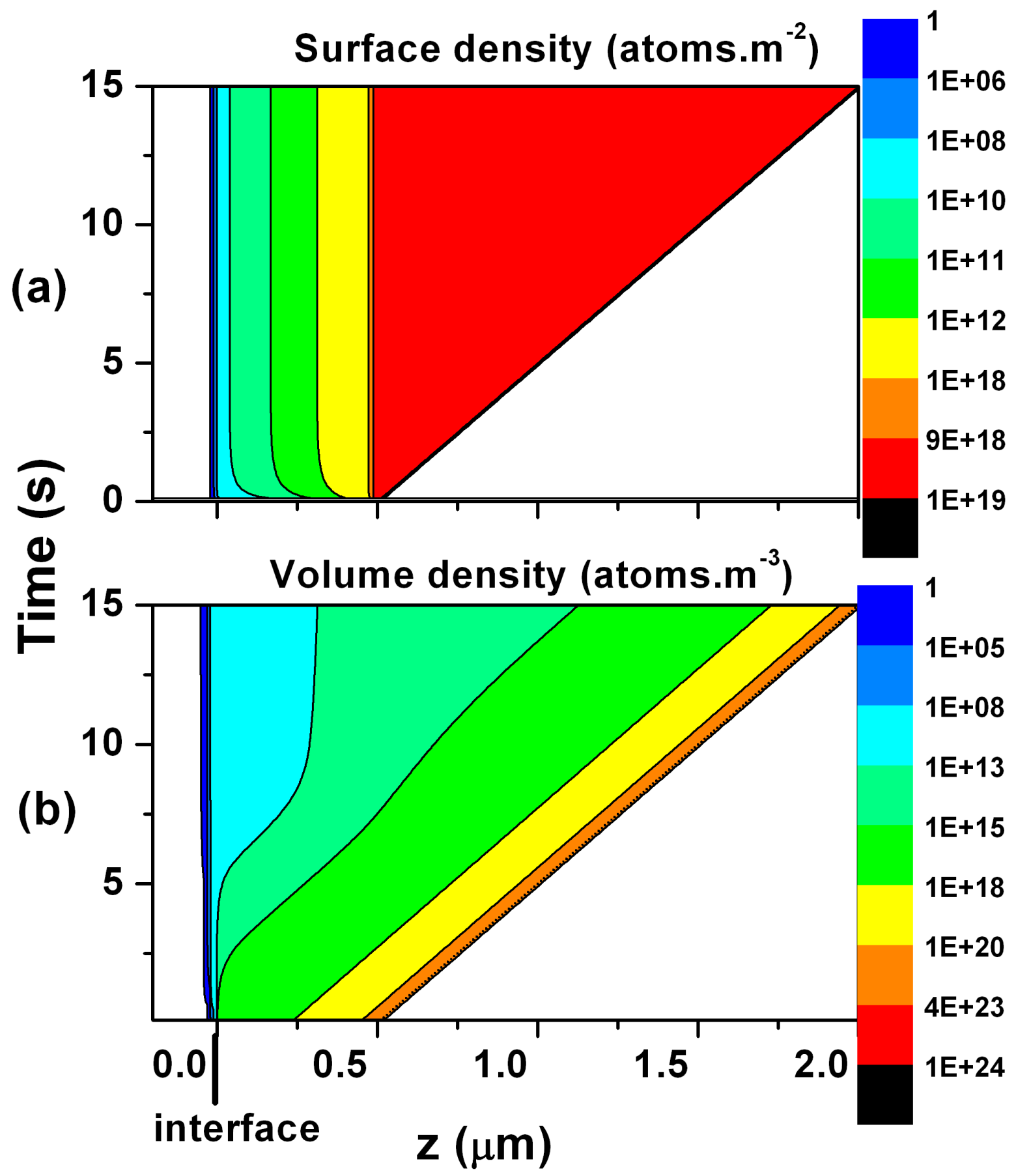


Table 1. The parameters used in porosity module.

\begin{tabular}{|c|c|c|c|c|c|}
\hline \multicolumn{3}{|c|}{ Surface } & \multicolumn{3}{|c|}{ Bulk } \\
\hline Parameters & Region I & Region III & Parameters & Region II & Region IV \\
\hline$\omega_{0}\left(s^{-1}\right)$ & $10^{13}$ & $10^{13}$ & $\omega_{0}\left(s^{-1}\right)$ & $10^{13}$ & $10^{13}$ \\
\hline$L_{S D}(\mathrm{~nm})$ & 0.5 & 3.46 & $L_{\text {trap }}(\mathrm{nm})$ & 0.8 & 10.0 \\
\hline$E_{S D}(\mathrm{eV})$ & 1.0 & 0.9 & $E_{\text {trap }}(\mathrm{eV})$ & 1.2 & 2.6 \\
\hline$L_{\text {void }}(\mathrm{nm})$ & 100 & 100 & & & \\
\hline$E_{D}(\mathrm{eV})$ & 2.0 & 1.91 & & & \\
\hline$E_{H D}(\mathrm{eV})$ & 1.91 & 1.91 & & & \\
\hline$E_{\text {trap }}(\mathrm{eV})$ & 2.6 & 2.6 & & & \\
\hline$E^{I-I I I}(\mathrm{eV})$ & & 1.0 & & & \\
\hline$E^{I I I-I}(e V)$ & & 1.0 & & & \\
\hline
\end{tabular}

Table 2. The parameters used in porosity module.

\begin{tabular}{llll}
\hline \multicolumn{2}{c}{ Co-deposited layer } & \multicolumn{2}{c}{ Target } \\
\hline Parameters & values & Parameters & values \\
\hline$E^{I-I I}(\mathrm{eV})$ & 2.6 & $E^{I I I-I V}(\mathrm{eV})$ & 2.6 \\
$f_{\text {imp }, c d}$ & 0.11 & $f_{\text {imp }, t}$ & 0.10 \\
$S_{I} / V_{I I}(\mathrm{~m})$ & $10^{8}$ & $S_{I I I} / V_{I V}(\mathrm{~m})$ & $10^{8}$ \\
$\sigma_{\text {Trap }, t=0}^{I}\left(m^{-2}\right)$ & $10^{19}$ & $\sigma_{\text {Trap }, t=0}^{I I I}\left(m^{-2}\right)$ & $10^{19}$ \\
\hline
\end{tabular}

